\title{
Heavy dark matter particle annihilation in dwarf spheroidal galaxies: Radio signals at the SKA telescope
}

\author{
Arpan Kar, ${ }^{1, *}$ Sourav Mitra, ${ }^{2}$ Biswarup Mukhopadhyaya, ${ }^{1}$ and Tirthankar Roy Choudhury $\odot^{3}$ \\ ${ }^{1}$ Regional Centre for Accelerator-based Particle Physics, Harish-Chandra Research Institute, HBNI, \\ Chhatnag Road, Jhunsi, Allahabad-211 019, India \\ ${ }^{2}$ Surendranath College, 24/2 M. G. Road, Kolkata, West Bengal 700009, India \\ ${ }^{3}$ National Centre for Radio Astrophysics, TIFR, Post Bag 3, Ganeshkhind, Pune 411007, India
}

(Received 21 September 2019; published 27 January 2020)

\begin{abstract}
A weakly interacting dark matter candidate is difficult to detect at high-energy colliders like the LHC, if its mass is close to or higher than a TeV. On the other hand, pair annihilation of such particles may give rise to $e^{+} e^{-}$pairs in dwarf spheroidal galaxies ( $\mathrm{dSph}$ ), which in turn can lead to radio synchrotron signals that are detectable at the upcoming Square Kilometre Array (SKA) telescope within a moderate observation time. We investigate the circumstances under which this complementarity between collider and radio signals of dark matter can be useful in probing physics beyond the standard model of elementary particles. Both particle physics issues and the roles of diffusion and electromagnetic energy loss of the $e^{ \pm}$are taken into account. First, the criteria for detectability of trans-TeV dark matter are analyzed independently of the particle physics model(s) involved. We thereafter use some benchmarks based on a popular scenario, namely, the minimal supersymmetric standard model. It is thus shown that the radio flux from a dSph like Draco should be observable in about $100 \mathrm{~h}$ at the SKA, for dark matter masses up to 4-8 TeV. In addition, the regions in the space spanned by astrophysical parameters, for which such signals should be detectable at the SKA, are marked out.
\end{abstract}

DOI: 10.1103/PhysRevD.101.023015

\section{INTRODUCTION}

Some yet unseen weakly interacting massive particles (WIMPs) are often thought of as constituents of dark matter (DM) in our Universe. Many scenarios beyond the standard model (SM) of particle physics including them are regularly proposed, with various phenomenological implications. They are constrained by the data on the relic density [1] of the Universe as well as various direct search experiments $[2,3]$. It is expected that a WIMP DM candidate should also be detected at the LHC in the form of missing transverse energy (see, for example, Refs. [4-6]).

Detectability at colliders and also in direct search experiments, however, depends on the mass as well as the interaction cross section of the DM particle. In particular, detection becomes rather difficult if the WIMP mass approaches a $\mathrm{TeV}$ [7]. For a weakly interacting DM, production at the LHC has to depend mostly on DrellYan processes in which the rate gets suppressed by the $s$, the

\footnotetext{
*arpankar@hri.res.in
}

Published by the American Physical Society under the terms of the Creative Commons Attribution 4.0 International license. Further distribution of this work must maintain attribution to the author(s) and the published article's title, journal citation, and DOI. Funded by SCOAP ${ }^{3}$. square of the subprocess center-of-mass energy, and also by the parton distribution function at high $x$. An exception is the minimal supersymmetric standard model (MSSM) [8]; there, the production of colored superparticles (squarks/ gluions) via strong interaction may be followed by decays in cascade leading to DM pair production. However, here, too, the event rates go down drastically when one looks at the current constraints on colored superparticle masses as well as the various other limits on the MSSM spectrum. On the whole, while a near-TeV DM particle still admits of some hope at the LHC in the MSSM [7,9-11], the reach is considerably lower for most other scenarios in which the "dark sector" is at most weakly interacting $[12,13]$. It is therefore a challenge to think of additional indirect evidence, if a trans-TeV DM particle has to be explored.

The annihilation of DM particles in our galaxy as well as in extra-galactic objects leads to gamma-ray signals [14-20] as well as positrons, antiprotons, etc. [21-24]. Constraints have been imposed on DM annihilation rates in various ways out of the (non)observation of such signals [25-28]. An alternative avenue to explore is that opened by radio synchrotron emission from galaxies, arising out of electron-positron pairs generated from DM annihilation [29-32]. In this paper, we focus on the potential of the upcoming Square Kilometre Array (SKA) radio telescope [33] in this regard. 
While the prospect of radio fluxes unveiling DM annihilation has been explored in earlier works [2932,34-36], it was pointed out in Ref. [37] that SKA opens up a rather striking possibility. The annihilation of trans$\mathrm{TeV}$ DM pairs in dwarf spheroidal galaxies (dSph) lead to electron-positron pairs, which, upon acceleration by the galactic magnetic field, produces such radio synchrotron emission. DSphs are suitable for studying DM, since star formation rates there are low $[38,39]$, thus minimizing the possibility of signal originating from astrophysical processes. Their generic faintness prompts one to concentrate on such galaxies which are satellites of the Milky Way. The SKA can ensure sufficient sensitivity required to detect the faint signal from the sources and, at the same time, will have high enough resolution to remove the foregrounds. It was shown in Ref. [37] that about $100 \mathrm{~h}$ of observation at the SKA can take us above the detectability threshold for radio signals from the annihilation of DM particles in the 5-10 TeV range. Of course, the compatibility of such massive WIMPs with the observed relic density requires a dark sector spectrum with enough scope for coannihilation in the early Universe, as was demonstrated in Ref. [37] in the context of the MSSM. What one learns from such an exercise is that the probe of at least some DM scenarios should thus be possible on a timescale comparable with the running period of the LHC and that the reach of the LHC [7,9-11] for WIMP detection may be exceeded considerably through such a probe. It was also found that cases in which SKA could observe radio fluxes from a dSph were consistent with limits from $\gamma$-ray observations as well as antiparticles in cosmic rays.

To ascertain which scenarios are more accessible in such radio probes, one needs to understand in detail the mechanisms whereby high-mass DM particles can produce higher radio fluxes and also the effects of astrophysical processes that inevitably affect radio emission. This is the task undertaken in the present work.

The spectrum as well as the dynamics of the particle physics scenario, along with the DM profile in a dSph, is responsible for DM annihilation as well as the subsequent cascades leading to electron-positron pairs. ${ }^{1}$ The electron (positron) energy distribution at the source function level is also the determined by the above factors [40-42]. However, they subsequently pass through the interstellar medium (ISM) of the galaxy, facing several additional effects. These include diffusion as well as electromagnetic energy loss in various forms, including the inverse Compton effect, synchrotron loss, Coulomb effect, and Bremsstrahlung

\footnotetext{
${ }^{1}$ In principle, electron-positron pairs may also be directly produced in a complete model-independent scenario. We have referred to cascade since our benchmark corresponds to $b \bar{b}, t \bar{t}$, $W^{+} W^{-}$, and $\tau^{+} \tau^{-}$as dominant annihilation channels, inspired by the MSSM in which direct $e^{+} e^{-}$is not found to dominate for high DM masses.
}

effect $[40,41,43,44]$. Besides, the galactic magnetic field is operative all along. The way these affect the final $e^{+} e^{-}$ energy distribution is highly interconnected and nonlinear. For example, while the magnetic field causes electrons to lose more energy in synchrotron radiation, it puts a check on the reduction of the flux through diffusion by confining them longer within the periphery of the $\mathrm{dSph}$. This check applies to electrons at lower energies at the cost of those at higher energies. Also, electromagnetic energy loss enhances the population of low-energy electrons and positrons, the enhancement being more when they have higher kinematic limits, enabled by the higher mass of the DM particle. Such low-energy $e^{+} e^{-}$pairs enhance the flux in the frequency range appropriate for a radio telescope.

In the following sections, we analyze the various ingredients in the radio flux generation process, as outlined above. We first do this for fixed DM particle masses and for values of their annihilation cross sections fixed by hand. The relative strengths of the effects of the particle theoretical scenario as well as diffusion and radiative process are thus assessed. This also serves to evolve an understanding of the dependence on the diffusion coefficient; parameters involved in electromagnetic energy loss; and, of course, the strength of the galactic magnetic field. If the nature of the DM particle(s) is known in independent channels, then the observation of the observed radio flux may be turned around to improve our understanding of these astrophysical parameters, using the results presented here.

Finally, we use some theoretical benchmark points to demonstrate the usefulness of the SKA in probing transTeV DM. A few sample MSSM spectra are used, largely because they offer the scope of coannihilation that is so essential for maintaining the right relic density [37]. Using the minimum annihilation cross section required for any DM mass for detection at SKA (in $100 \mathrm{~h}$ ) and the maximum value of this cross section that is compatible with limits from $\gamma$-ray and cosmic-ray data, we show that several benchmark points with DM mass in the $1-8 \mathrm{TeV}$ range, which are yet to be ruled out by any observation, can be investigated in $100 \mathrm{~h}$ of SKA observation.

We have organized the paper as follows. In Sec. II, we discuss in a somewhat brief manner the calculations of synchrotron flux originating from DM annihilation inside a dSph. In Sec. III, we analyze the effects of various astrophysical parameters in different steps of the production of radio flux. Section IV describes the features of heavy DM. In Sec. V, we show the detectability curves or threshold limits for observing radio flux in SKA, in both model-independent as well as model-dependent ways. We also show the final radio fluxes for some theoretical benchmarks. Finally, in Sec. VI, we conclude.

\section{ESSENTIAL PROCESSES: RECAPITULATION}

We start with a brief resume of the sequence of processes that leads to radio flux from a dSph, as discussed, for 
example, in Refs. [30,31,40,41,43,44]. Dark matter pair annihilation inside of a dSph can produce SM particle pairs such as $b \bar{b}, \tau^{+} \tau^{-}, W^{+} W^{-}, t \bar{t}$, etc. These particles then cascade and give rise to large amount of $e^{ \pm}$flux, the resulting energy distribution of which can be obtained from the source function $[41,42]$,

$$
Q_{e}(E, r)=\langle\sigma v\rangle\left\{\sum_{f} \frac{d N_{f}^{e}(E)}{d E} B_{f}\right\} N_{\text {pairs }}(r),
$$

where $\langle\sigma v\rangle$ and $N_{\text {pairs }}(r)\left(=\frac{\rho_{\chi}^{2}(r)}{2 m_{\chi}^{2}}\right)$ are, respectively, the velocity-averaged annihilation rate and the number density of DM pairs inside the dSph. $m_{\chi}$ is the DM mass, and $\rho_{\chi}(r)$ is the DM density profile in the $\mathrm{dSph}$ as a function of radial distance $r$ from the center of the dSph. For our analysis, we have taken Draco ${ }^{2} \mathrm{dSph}$ assuming an Navarro-Frenk-White (NFW) profile [48], with

$$
\rho_{\chi}(r)=\frac{\rho_{s}}{\left(\frac{r}{r_{s}}\right)\left(1+\frac{r}{r_{s}}\right)^{2}},
$$

where $\rho_{s}=1.4 \mathrm{GeV} . \mathrm{cm}^{-3}$ and $r_{s}=1.0 \mathrm{kpc}[41,44]$. $\frac{d N_{f}^{e}(E)}{d E} B_{f}$ estimates the number of $e^{ \pm}$produced with energy $E$ per annihilation in any of the aforementioned SM channels $(f)$ having branching fraction $B_{f}$.

Figure 1 shows the electron energy distribution for four different annihilation channels $\left(b \bar{b}, \tau^{+} \tau^{-}, W^{+} W^{-}, t \bar{t}\right)$ and for two DM masses, namely, $m_{\chi}=300 \mathrm{GeV}$ (upper left panel) and $5 \mathrm{TeV}$ (upper right panel). For all cases, $\langle\sigma v\rangle$ has been assigned a fixed value $\left(10^{-26} \mathrm{~cm}^{3} \mathrm{~s}^{-1}\right)$, and the branching fraction corresponding to each channel has been set in turn at $100 \%$. In addition, a comparison between cases with the two masses has also been shown in the lower panel for the $b \bar{b}$ and $\tau^{+} \tau^{-}$channels. The prediction for $t \bar{t}$ and $W^{+} W^{-}$falls in between those curves. All these energy distributions are obtained using MICROMEGAs [49,50]. Further discussions on these curves will be taken up in Sec. IV.

The electrons produced in DM pair annihilation diffuse through the ISM in the galaxy and lose energy through various electromagnetic processes. Assuming a steady-state and homogeneous diffusion, the equilibrium distribution $\frac{d n}{d E}$ can be obtained by solving the equation $[31,40,41,43,44,51]$

$D(E) \nabla^{2}\left(\frac{d n}{d E}\right)+\frac{\partial}{\partial E}\left(b(E) \frac{d n}{d E}\right)+Q_{e}(E, r)=0$,

where $D(E)$ is the diffusion parameter. The exact form of $D(E)$ is not known for dSphs; hence, for simplicity, we

\footnotetext{
${ }^{2}$ In this paper, we have used the dSph Draco to illustrate our points, primarily because the relevant parameters such as the $J$ factor are somewhat better constrained for this object [45]. However, similar conclusions apply to other dSphs such as Seg1, Carina, Fornax, Sculptor, etc. [45-47].
}

assume it to have a Kolmogorov spectrum $D(E)=$ $D_{0}\left(\frac{E}{\mathrm{GeV}}\right)^{0.3}$, where $D_{0}$ is the diffusion coefficient $[32,41,44]$. Very little is known about the value of $D_{0}$ for dSphs due to their very low luminosity. A choice like $D_{0}=$ $3 \times 10^{28} \mathrm{~cm}^{2} \mathrm{~s}^{-1}$ is by and large reasonable for a dSph such as Draco [44], although higher values of $D_{0}$, too, are sometimes used as a benchmark [36]. We have mostly used $D_{0}=3 \times 10^{28} \mathrm{~cm}^{2} \mathrm{~s}^{-1}$ and demonstrated side by side the effect of other values of this largely unknown parameter. As explained in Refs. [30,31,52], assuming a proper scaling, $D_{0}$ for a dSph can also in principle have a value one order lower than that in the Milky Way [53]. However, the present study is not restricted to this value, mainly $D_{0} \lesssim 3 \times 10^{28} \mathrm{~cm}^{2} \mathrm{~s}^{-1}$. The detectability of the radio flux for higher $D_{0}$, too, has been studied by us, as will be seen when we come to Fig. 13 .

The energy loss term $b(E)$ takes into account all the electromagnetic energy loss processes such as the inverse Compton (IC) effect, synchrotron (Synch) radiation, Coulomb loss (Coul), bremsstrahlung (Brem), etc., and their combined effect can be expressed as $[40,41,43]$

$$
\begin{aligned}
b(E)= & b_{\mathrm{IC}}^{0}\left(\frac{E}{\mathrm{GeV}}\right)^{2}+b_{\mathrm{Synch}}^{0}\left(\frac{E}{\mathrm{GeV}}\right)^{2}\left(\frac{B}{\mu G}\right)^{2} \\
& +b_{\mathrm{Coul}}^{0} n_{e}\left[1+\frac{\log \left(\frac{E / m_{e}}{n_{e}}\right)}{75}\right] \\
& +b_{\mathrm{Brem}}^{0} n_{e}\left[\log \left(\frac{E / m_{e}}{n_{e}}\right)+0.36\right],
\end{aligned}
$$

where $m_{e}$ is the electron mass and $n_{e}$ is the average thermal electron density in the $\mathrm{dSph}$ (value of $n_{e} \approx 10^{-6}$ ). Values of the energy loss coefficients are taken to be $b_{\mathrm{IC}}^{0} \simeq 0.25$, $b_{\text {Synch }}^{0} \simeq 0.0254$, and $b_{\text {Coul }}^{0} \simeq 6.13 b_{\mathrm{Brem}}^{0} \simeq 1.51$, all in units of $10^{-16} \mathrm{GeV} \mathrm{s}^{-1}[40,41,43]$. In a dSph like Draco, the first two terms (i.e., IC and Synch) in Eq. (4) dominate over the last two terms (i.e., Coul and Brem) for $E>1 \mathrm{GeV}$ [31]. The energy loss term $b(E)$ depends on the galactic magnetic field $(B)$ through the synchrotron loss term, which goes as $B^{2}$. It is extremely difficult to get insight (say, through polarization experiments) for the magnetic field properties of dSph. The lack of any strong observational evidence suggests that the magnetic fields could be, in principle, extremely low. On the other hand, there could be various effects which can significantly contribute to the magnetic field strengths in $\mathrm{dSph}$. In fact, numerous theoretical arguments have been proposed for values of $B$ at the $\mu \mathrm{G}$ level. It has been argued, for example, that the observed fall of $B$ from the center of the Milky Way to its peripheral region can be linearly extrapolated to nearby $\mathrm{dSphs}$, leading to $B \gtrsim 1 \mu \mathrm{G}$. The possibility of a dSph having its own magnetic field has also been suggested. For detailed discussions, we refer the reader to Ref. [54]. For the dSph considered in this work, we have mostly used $B=1 \mu \mathrm{G}[32,41,44]$, but more conservative values like 

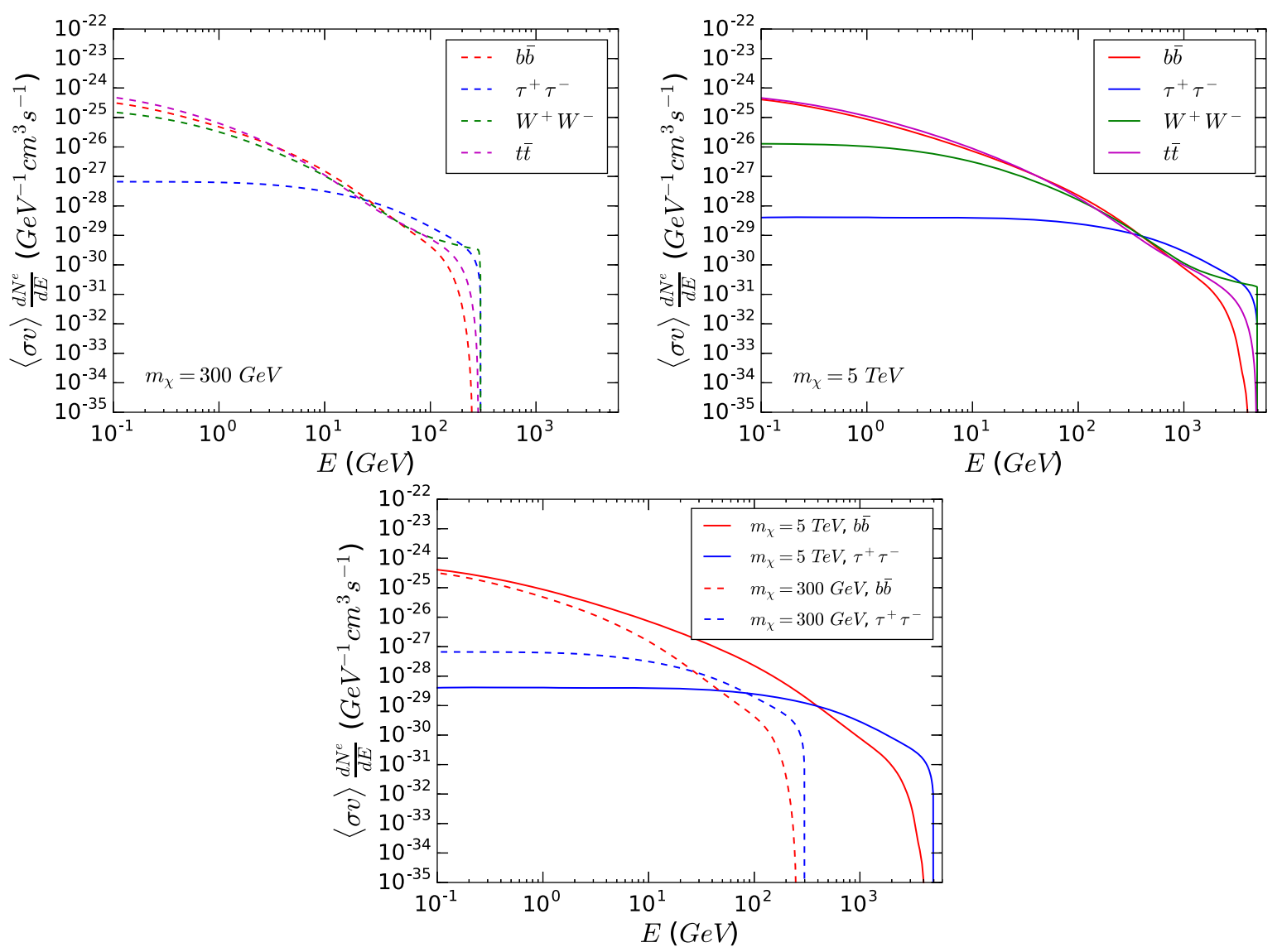

FIG. 1. Upper panel: Source functions per unit annihilation $\left(\langle\sigma v\rangle \frac{d N^{e}}{d E}\right)$ vs electron energy $(E)$ in different annihilation channels for two DM masses, $300 \mathrm{GeV}$ (upper left panel) and $5 \mathrm{TeV}$ (upper right panel). Lower panel: Comparison of the same for two DM masses, $300 \mathrm{GeV}$ (dashed curves) and $5 \mathrm{TeV}$ (solid curves) in two annihilation channels, $b \bar{b}$ (red lines) and $\tau^{+} \tau^{-}$(blue lines). The annihilation rate for each panel is $\langle\sigma v\rangle=10^{-26} \mathrm{~cm}^{3} \mathrm{~s}^{-1}$.

$B \sim 10^{-1}-10^{-3} \mu \mathrm{G}$ have also been considered. The manner in which diffusion and electromagnetic processes affect our observables will be discussed in detail in Secs. III and $\mathrm{V}$. The overall potential of the SKA in probing regions in the $D_{0}-B$ space in a correlated fashion will be reported in Sec. V.

Equation (3) has a solution of the form $[31,40,41,44]$

$$
\frac{d n}{d E}(r, E)=\frac{1}{b(E)} \int_{E}^{m_{\varkappa}} d E^{\prime} G(r, \Delta v) Q_{e}\left(E^{\prime}, r\right),
$$

with the boundary condition $\frac{d n}{d E}\left(r_{h}\right)=0$, where $r_{h}$ is the radius of the diffusion zone. For Draco, $r_{h}$ is taken to be $2.5 \mathrm{kpc}[41,44] . G(r, \Delta v)$ is the Green's function of the equation and has a form

$$
\begin{aligned}
G(r, \Delta v)= & \frac{1}{\sqrt{4 \pi \Delta v}} \sum_{n=-\infty}^{n=\infty}(-1)^{n} \int_{0}^{r_{h}} d r^{\prime} \frac{r^{\prime}}{r_{n}}\left(\frac{\rho_{\chi}\left(r^{\prime}\right)}{\rho_{\chi}(r)}\right)^{2} \\
& \times\left[\exp \left(-\frac{\left(r^{\prime}-r_{n}\right)^{2}}{4 \Delta v}\right)-\exp \left(-\frac{\left(r^{\prime}+r_{n}\right)^{2}}{4 \Delta v}\right)\right]
\end{aligned}
$$

with $r_{n}=(-1)^{n} r+2 n r_{h} \cdot \sqrt{\Delta v}$ is called the diffusion length scale, expressed as

$$
\Delta v=\int_{E}^{E^{\prime}} d \tilde{E} \frac{D(\tilde{E})}{b(\tilde{E})}
$$

$\sqrt{\Delta v}$ determines the distance traveled by an electron as it loses energy from $E^{\prime}$ to $E$. For small galaxies like dSphs, the mean value of this length scale is expected to be larger than $r_{h}$ even for nonconservative choices of $D_{0}$ and $B$. We shall discuss this in detail in Sec. III. After interacting with the magnetic field $B$ present inside the galaxy, the produced electron/positron distribution $\frac{d n}{d E}$ will emit synchrotron radiation (with frequency $\nu$ ) at a rate governed by the synchrotron emission power $P_{\text {Synch }}(\nu, E, B)[40,41,43,44,51]$.

In Fig. 2, we have shown the dependence of the synchrotron emission power on the electron energy $E$ for two different magnetic fields $(B=1$ and $0.1 \mu \mathrm{G})$ and for two frequencies $\left(\nu=10\right.$ and $10^{4} \mathrm{MHz}$ ). For each frequency shown, it is clear that a stronger magnetic field always intensifies the power spectrum. 


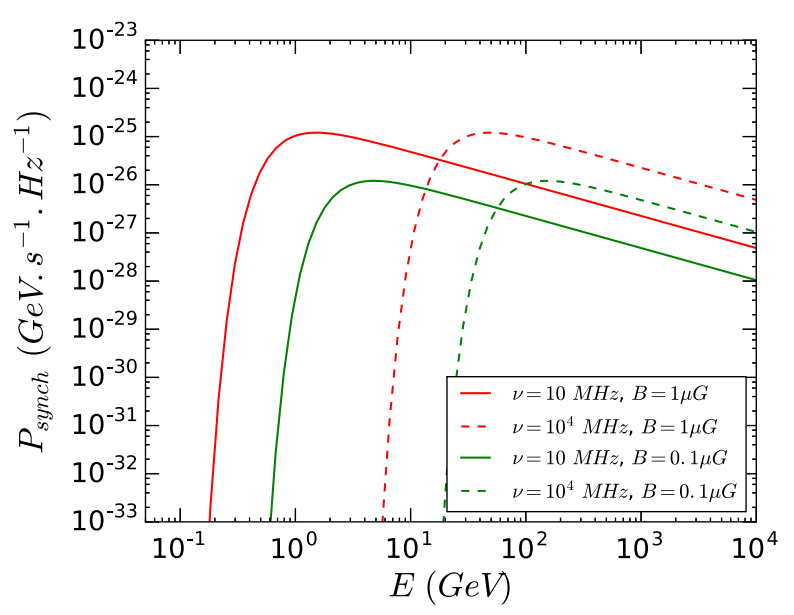

FIG. 2. Synchrotron power spectrum $\left(P_{\text {Synch }}\right)$ vs energy $(E)$ at two frequencies: 10 (solid lines) and $10^{4} \mathrm{MHz}$ (dashed lines), with two different magnetic fields, $B=1$ (red) and $0.1 \mu \mathrm{G}$ (green).

The synchrotron emissivity $J_{\text {Synch }}$ as a function of frequency $\nu$ and radius $r$ is obtained by folding $\frac{d n}{d E}(r, E)$ with $P_{\text {Synch }}(\nu, E, B)$ as

$$
J_{\text {Synch }}(\nu, r)=2 \int_{m_{e}}^{m_{\chi}} d E \frac{d n}{d E}(r, E) P_{\text {Synch }}(\nu, E, B) .
$$

Finally, the approximate radio synchrotron flux can be obtained by integrating $J_{\text {Synch }}$ over the diffusion size $\left(r_{h}\right)$ of the $\mathrm{dSph}[40,41,43,44,51]$

$$
S_{\nu}(\nu)=\frac{1}{L^{2}} \int d r r^{2} J_{\operatorname{Synch}}(\nu, r),
$$

where $L$ is the luminosity distance of the $\mathrm{dSph}$. For Draco, $L \sim 80 \mathrm{kpc}[41,45])$.

\section{EFFECT OF VARIOUS ASTROPHYSICAL PARAMETERS}

Let us now take a closer look at the astrophysical effects encapsulated in Eqs. (3) and (4). Such effects are driven by the diffusion coefficient $D_{0}$ and the electromagnetic energy loss coefficient $b(E)$, which determine the steady-state $e^{ \pm}$ distribution $\frac{d n}{d E}(r, E)$ for a given $\frac{d N^{e}}{d E}$. As has been already mentioned, $b(E)$ is implicitly dependent on the galactic magnetic field $B$.

We use again the two DM masses $\left(m_{\chi}=300 \mathrm{GeV}\right.$ and $5 \mathrm{TeV}$ ) for studying the effects of $D_{0}$ and $b(E)$. To have some idea about these effects separately, as well as their contribution in an entangled fashion, we have considered the implication of Eq. (3) for three different scenarios:

(i) NSD (neglecting spatial diffusion): considering only the effect of energy loss term $b(E)$ and neglecting the spatial diffusion $D(E)$ [i.e., solution of Eq. (3) by setting $D(E)=0$ ].

(ii) $\mathrm{Nb}$ (neglecting $b(E)$ ): considering the effect of diffusion parameter $D_{0}$ and neglecting the energy loss term $b(E)$ [i.e., solution of Eq. (3) by setting $b(E)=0]$.

(iii) $\mathrm{SD}+\mathrm{b}($ Spatial Diffusion $+b(E)$ ): considering the effects of both the diffusion parameter $D_{0}$ and the energy loss term $b(E)$ [i.e., the complete solution as explained in Eq. (5)].

For the NSD scenario, the solution of Eq. (3) has a simpler form [40]:

$$
\left.\frac{d n}{d E}(r, E)\right|_{\mathrm{NSD}}=\frac{1}{b(E)} \int_{E}^{m_{\chi}} d E^{\prime} Q_{e}\left(E^{\prime}, r\right) .
$$

Note that $b(E)$ increases with $E$ [from Eq. (4)]. Its effect on the number density in different energy regions is more prominent for this NSD scenario in which we neglect the effect of distribution. The number density decreases in the high-energy region due to the combined effect of the $\frac{1}{b(E)}$ suppression as well as the lower limit of the integration in Eq. (10). This can also be seen from Fig. 3, in which we have plotted the ratio of $\frac{d n}{d E}$ and the source function $Q_{e}$ (which determines the initial electron flux due to DM annihilation) against $E$. The term $\frac{d n}{d E} \frac{1}{Q_{e}}$ (in the unit of s${ }^{-1}$ ) essentially determines how the shape of the initial distribution $Q_{e}$ gets modified due to the effect of various astrophysical processes. As discussed in Sec. II, the energy loss term $b(E)$ depends on the square of the magnetic field $B$ through synchrotron loss. Thus, $b(E)$ increases with the increase of $B$, which in turn reduces the $\frac{d n}{d E}$ at all $E$. This phenomenon can also be observed in Fig. 3, in which the red and magenta lines indicate the distributions for $B=1$ and $10 \mu \mathrm{G},{ }^{3}$ respectively. For comparison, we have also shown the cases for $B=0.1 \mu \mathrm{G}$ (green lines). Note that the latter case coincides with the case for $B=1 \mu \mathrm{G}$. This is due to the fact that the magnetic field dependence (through synchrotron loss) in the energy loss term $b(E)$ gets suppressed by the inverse Compton $\left(b_{\mathrm{IC}}\right)$ term for lower $B$ $(B<1 \mu \mathrm{G})$, which can be clearly seen from Eq. (4). Also, for heavier DM masses, the energetic electrons are produced in greater abundance (as seen from Fig. 1), which can lead to a larger $\frac{d n}{d E} \frac{1}{Q_{e}}$. The effects of two different DM masses, $m_{\chi}=300 \mathrm{GeV}$ and $5 \mathrm{TeV}$, are shown in this figure by dashed and solid lines, respectively.

\footnotetext{
${ }^{3} B=10 \mu \mathrm{G}$ has been used in this figure for the sake of comparison with $1 \mu \mathrm{G}$, just to see the effect of "large $B$." In our prediction on observable radio flux, more conservative (and perhaps realistic) values of $B$ have been used.
} 


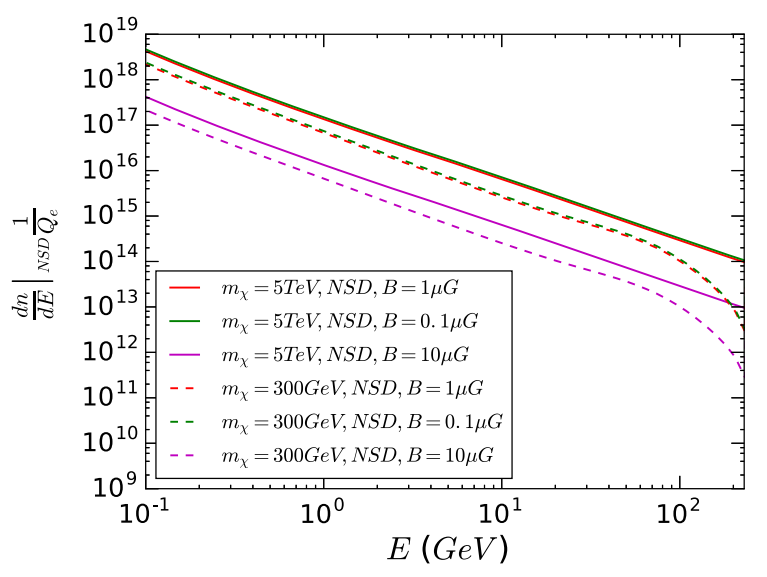

FIG. 3. $\frac{d n}{d E} \frac{1}{Q_{e}}$ vs electron energy $E$ plot for two DM masses, $5 \mathrm{TeV}$ (solid lines) and $300 \mathrm{GeV}$ (dashed lines), with magnetic fields $B=1$ (red), 0.1 (green), and $10 \mu \mathrm{G}$ (magenta) in the scenario in which diffusion in the system has been neglected (NSD). The annihilation channel is $b \bar{b}$ with annihilation rate $\langle\sigma v\rangle=10^{-26} \mathrm{~cm}^{3} \mathrm{~s}^{-1}$.

Now, for the $\mathrm{Nb}$ scenario, the solution becomes

$$
\left.\frac{d n}{d E}(r, E)\right|_{\mathrm{Nb}}=\frac{1}{D(E)} f\left(r, r_{h}\right) Q_{e}(r, E)
$$

with the same boundary condition as what we assumed for Eq. (5). Here,

$f\left(r, r_{h}\right)=\int_{r^{\prime}=r}^{r_{h}} d r^{\prime}\left(\frac{1}{r^{\prime 2}}\right)\left\{\int_{\tilde{r}=0}^{r^{\prime}} d \tilde{r}^{2} \tilde{r}^{2}\left(\frac{\rho_{\chi}(\tilde{r})}{\rho_{\chi}(r)}\right)^{2}\right\}$.

Since the effect of $b(E)$ is absent in this case, the distribution $\frac{d n}{d E}$ will not depend on the magnetic field $B$. Figure 4 shows the comparison between the NSD case (cyan lines) and the $\mathrm{Nb}$ case (black lines) at two different radii $(r=0.1 \mathrm{kpc}$, left panel, and $r=2 \mathrm{kpc}$, right panel) for $B=1 \mu \mathrm{G}$ and $D_{0}=3 \times 10^{28} \mathrm{~cm}^{2} \mathrm{~s}^{-1}$. It is clear from Eq. (11) that the ratio $\frac{d n}{d E} \frac{1}{Q_{e}}$ for the $\mathrm{Nb}$ scenario will not depend on the initial spectrum $Q_{e}$ and its energy profile will follow the energy dependence of $\frac{1}{D(E)}$. This presence of $D(E)$ in the denominator leads to $\frac{d n}{d E} \frac{1}{Q_{e}} \sim E^{-0.3}$ in the limit $b(E) \rightarrow 0$. This can also be verified from the relatively flat curves for $\mathrm{Nb}$ cases in this figure.

We should mention here that neglecting diffusion is not a bad assumption when the length scale $(\sqrt{\Delta v})$ over which the $e^{ \pm}$loses energy is much shorter than the typical size of the system $[40,41]$. However, for smaller systems like dSphs, the effect of diffusion cannot be neglected. This can be justified from Fig. 4, in which we have plotted the $\mathrm{SD}+\mathrm{b}$ case (i.e., taking diffusion into account along with energy loss effect, shown by red curves) along with the two previously mentioned scenarios. It is clear that the NSD scenario is strikingly different from the one which takes diffusion into account. The addition of diffusion in the system essentially suppresses the $e^{ \pm}$distribution, especially in the low-energy region. As a result, the plots for the $\mathrm{SD}+\mathrm{b}$ case closely match the $\mathrm{Nb}$ one at lower energy, but diverges following the NSD scenario at higher energies at which the effect of $b(E)$ dominates. This phenomenon can also be explained in terms of the diffusion length scale $(\sqrt{\Delta v})$, which is a result of combined effects of diffusion and energy loss [Eq. (7)]. It is indicative of the length covered by an electron as it loses energy from the source energy $E^{\prime}$ to the interaction energy $E$, which is typically larger than the size of the $\mathrm{dSph}$. To determine the minimum energy that an electron can possess before escaping the diffusion radius, we have plotted the interaction energy $E$ vs $\sqrt{\Delta v}$ in Fig. 5 for

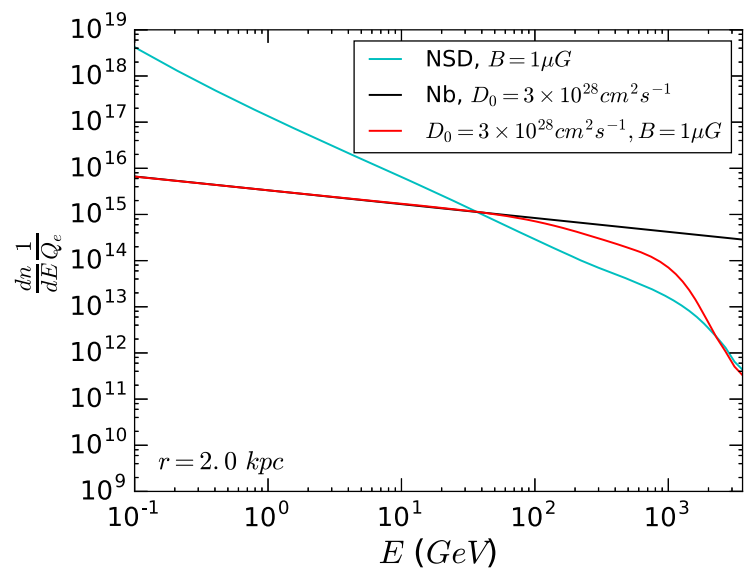

FIG. 4. $\frac{d n}{d E} \frac{1}{Q_{e}}$ vs electron energy $E$ at two different radii, $r=0.1 \mathrm{kpc}$ (left panel) and $r=2.0 \mathrm{kpc}$ ( right panel) for $m_{\chi}=5 \mathrm{TeV}$ in three scenarios, NSD, $\mathrm{Nb}$, and SD + b. Cases including diffusion $\left(\mathrm{Nb}\right.$ and $\mathrm{SD}+\mathrm{b}$ ) have $D_{0}=3 \times 10^{28} \mathrm{~cm}^{2} \mathrm{~s}^{-1}$, and cases including the energy loss effect (NSD and SD $+\mathrm{b}$ ) have magnetic field $B=1 \mu \mathrm{G}$. For all cases, the annihilation channel is $b \bar{b}$ with annihilation rate $\langle\sigma v\rangle=10^{-26} \mathrm{~cm}^{3} \mathrm{~s}^{-1}$. 

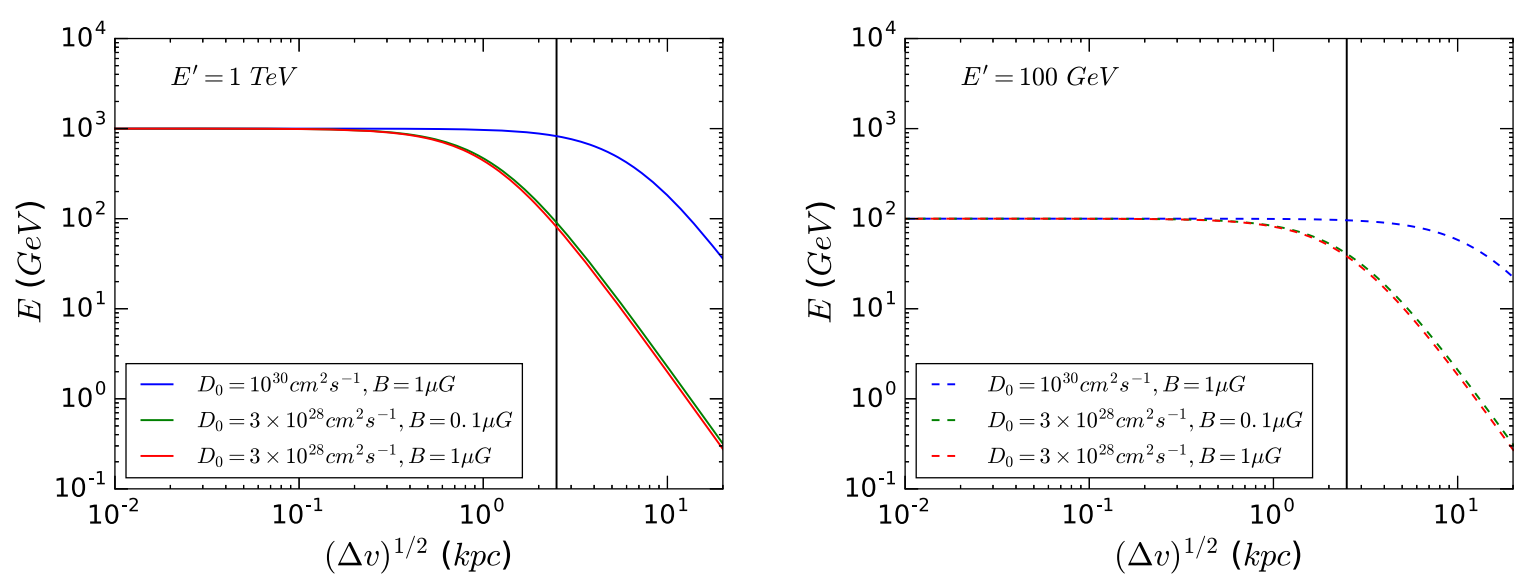

FIG. 5. Electron interaction energy $E(\mathrm{GeV})$ vs diffusion length scale $\sqrt{\Delta v}(\mathrm{kpc})$ for two different source energies, $E^{\prime}=1 \mathrm{TeV}$ (left panel) and $E^{\prime}=100 \mathrm{GeV}$ (right panel). The vertical black solid lines indicate the diffusion size of the galaxy $\left(r_{h}=2.5 \mathrm{kpc}\right)$. Here, three different sets of astrophysical parameters have been chosen, $D_{0}=3 \times 10^{28} \mathrm{~cm}^{2} \mathrm{~s}^{-1}, B=1 \mu \mathrm{G}(\mathrm{red}) ; D_{0}=10^{30} \mathrm{~cm}^{2} \mathrm{~s}^{-1}$, $B=1 \mu \mathrm{G}$ (blue); and $D_{0}=3 \times 10^{28} \mathrm{~cm}^{2} \mathrm{~s}^{-1}, B=0.1 \mu \mathrm{G}$ (green).

different sets of $D_{0}$ and $B$. The left and right panels correspond to the source energy $E^{\prime}=1 \mathrm{TeV}$ and $100 \mathrm{GeV}$, respectively. The diffusion radius (for Draco) $r_{h}=2.5 \mathrm{kpc}$ is shown by the black solid lines for both
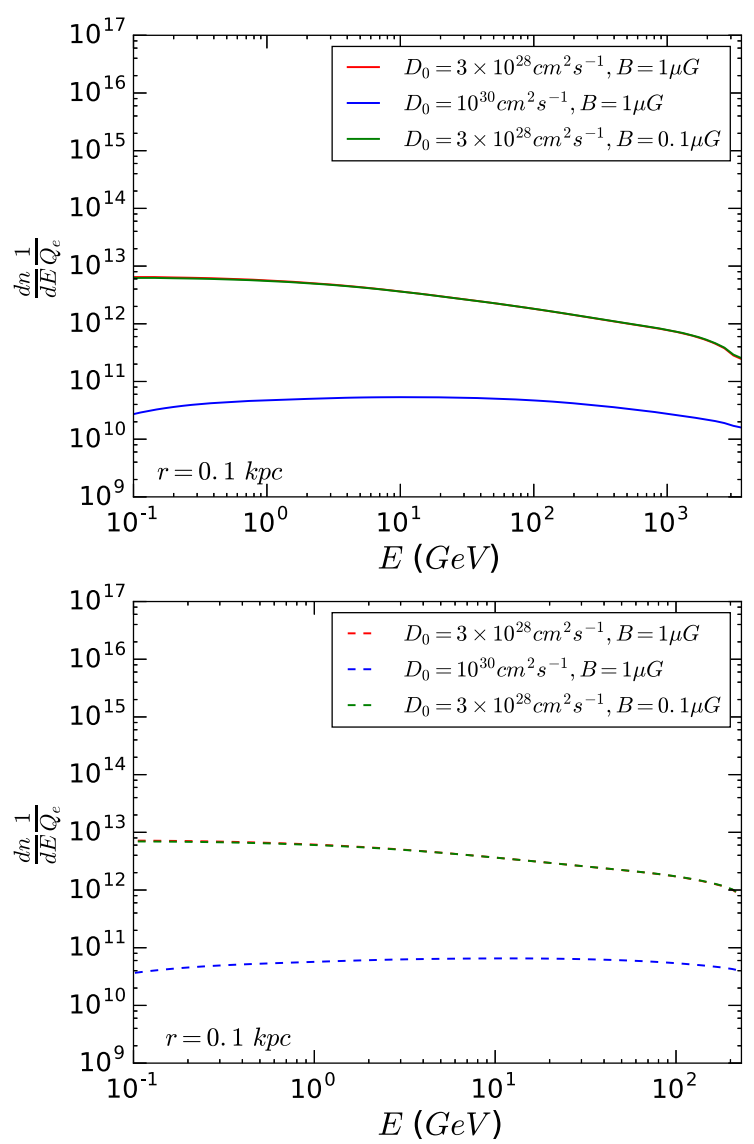

cases. The minimum energy is thus calculated from the point where the model crosses this line. A higher $D_{0}$ will increase this minimum value of interaction energy, and as a result, it reduces the $e^{ \pm}$number density by making them
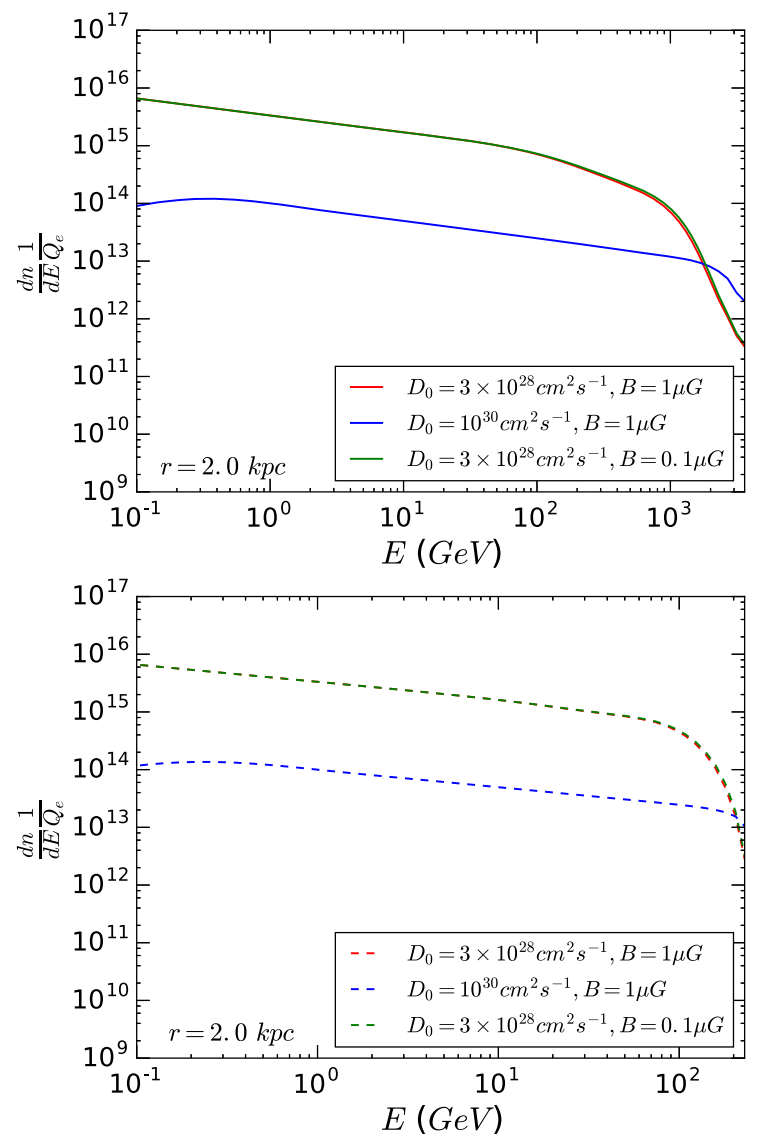

FIG. 6. $\frac{d n}{d E} \frac{1}{Q_{e}}$ vs electron energy $E$ at two different radii, $r=0.1 \mathrm{kpc}$ (left panel) and $r=2.0 \mathrm{kpc}$ (right panel) for two DM masses, $5 \mathrm{TeV}$ (upper panels, solid lines) and $300 \mathrm{GeV}$ (lower panels, dashed lines) in the $\mathrm{SD}+\mathrm{b}$ scenario. Astrophysical parameters considered here have been mentioned in the corresponding legends. For all cases, the annihilation channel is $b \bar{b}$ with annihilation rate $\langle\sigma v\rangle=10^{-26} \mathrm{~cm}^{3} \mathrm{~s}^{-1}$. 

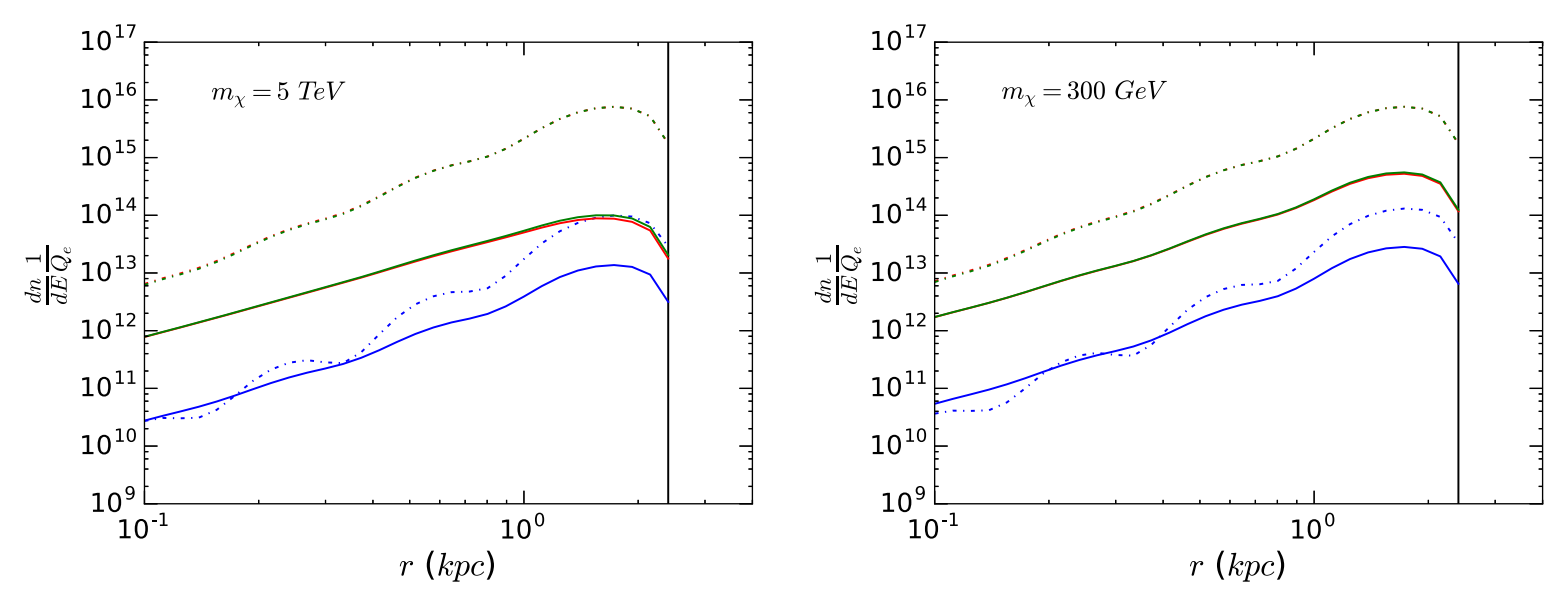

FIG. 7. Left panel: $\frac{d n}{d E} \frac{1}{Q_{e}}$ vs $r$ plot for $m_{\chi}=5 \mathrm{TeV}$ at two different electron energies, $E=0.1 \mathrm{GeV}$ (dashed dotted lines) and $E=1 \mathrm{TeV}$ (solid lines) in the SD $+\mathrm{b}$ scenario. The vertical black solid line indicates the diffusion size $\left(r_{h}\right)$ of the dSph. Three different sets of astrophysical parameters have been considered, $D_{0}=3 \times 10^{28} \mathrm{~cm}^{2} \mathrm{~s}^{-1}, B=1 \mu \mathrm{G}$ (red); $D_{0}=10^{30} \mathrm{~cm}^{2} \mathrm{~s}^{-1}, B=1 \mu \mathrm{G}$ (blue); and $D_{0}=3 \times 10^{28} \mathrm{~cm}^{2} \mathrm{~s}^{-1}, B=0.1 \mu \mathrm{G}$ (green). The annihilation channel is $b \bar{b}$ with annihilation rate $\langle\sigma v\rangle=10^{-26} \mathrm{~cm}^{3} \mathrm{~s}^{-1} \cdot R i g h t$ panel: Same as the left panel but at $E=0.1 \mathrm{GeV}$ (dashed dotted lines) and $E=100 \mathrm{GeV}$ (solid lines) for $m_{\chi}=300 \mathrm{GeV}$.

$\left(e^{ \pm}\right)$leave the diffusion zone earlier. Note that the cases with $B=0.1 \mu \mathrm{G}$ practically coincide with that for $B=1 \mu \mathrm{G}$. This is due to the fact that $b(E)$ remains unchanged for $B \leq 1 \mu \mathrm{G}$, as has already been mentioned. On the other hand, we have explicitly checked that a larger value of $B$ (say, $B=10 \mu \mathrm{G}$, not shown in the plot) will help the electron lose energy more quickly before escaping the diffusion zone, causing a suppression in the number density of high-energy electrons and an enhancement of the low-energy electrons.

Similar conclusions can also be drawn from Fig. 6. To illustrate the effects of $D_{0}$ and $B$ in the SD $+\mathrm{b}$ scenario, we have plotted $\frac{d n}{d E} \frac{1}{Q_{e}}$ against $E$ for the DM masses $5 \mathrm{TeV}$ (upper panels) and $300 \mathrm{GeV}$ (lower panels) at two different radii, 0.1 (left panels) and $2 \mathrm{kpc}$ (right panels). For all cases,

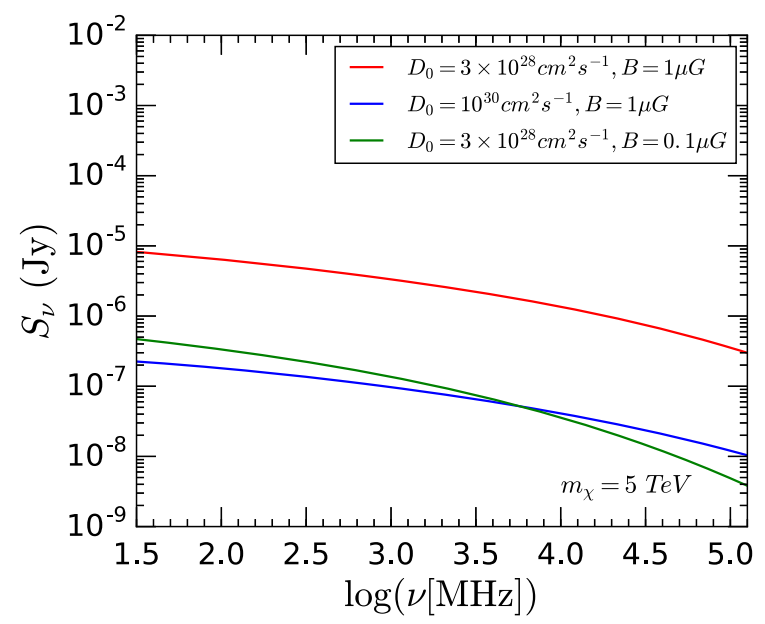

the dominant annihilation channel has been assumed to be $b \bar{b}$ with $\langle\sigma v\rangle=10^{-26} \mathrm{~cm}^{3} \mathrm{~s}^{-1}$. Cases with different $D_{0}$ and $B$ are indicated by different color coding as mentioned in the insets of corresponding figures. Similarly, Fig. 7 shows the variation of the same with respect to radius $r$ for those two DM masses $\left(m_{\chi}=5 \mathrm{TeV}\right.$-left panel and $m_{\chi}=300 \mathrm{GeV}$ right panel). For each DM mass, we have assumed two different combinations of energies, one being low ( $E=0.1 \mathrm{GeV}$ for both DM masses) and the other being high $\left(E=1 \mathrm{TeV}\right.$ for $m_{\chi}=5 \mathrm{TeV}$ and $E=100 \mathrm{GeV}$ for $\left.m_{\chi}=300 \mathrm{GeV}\right)$. In both panels, the black line indicates the diffusion radius $(2.5 \mathrm{kpc})$ for Draco. One can see that large $D_{0}$ (blue curves) implies a lower density distribution compared to the corresponding cases with a smaller diffusion coefficient. The difference is more prominent for low energy.

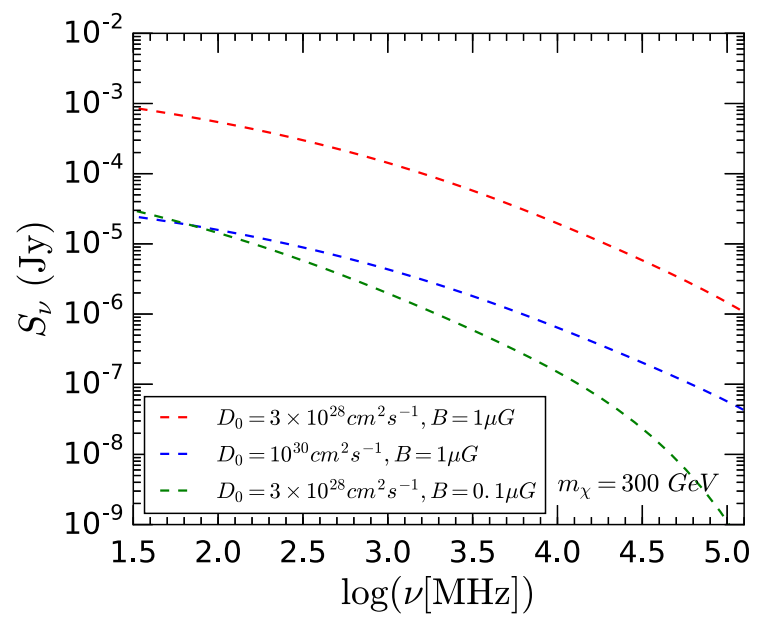

FIG. 8. Radio synchrotron flux $\left(S_{\nu}\right)$ vs frequency $(\nu)$ for two DM masses, $5 \mathrm{TeV}$ (left panel) and $300 \mathrm{GeV}$ (right panel) with different choices of astrophysical parameters $\left(D_{0}\right.$ and $\left.B\right)$ mentioned in the legends. The annihilation channel is $b \bar{b}$ with annihilation rate $\langle\sigma v\rangle=$ $10^{-26} \mathrm{~cm}^{3} \mathrm{~s}^{-1}$. 

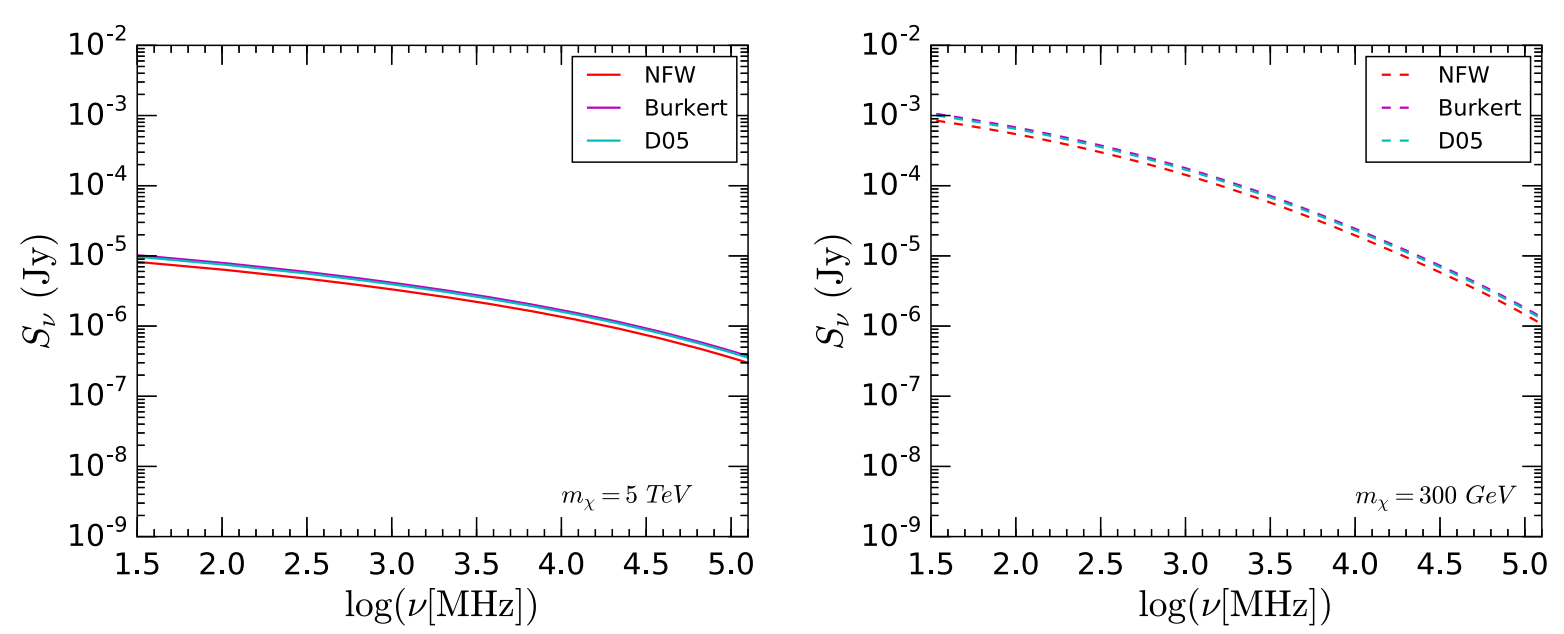

FIG. 9. Left panel: Radio synchrotron flux $S_{\nu}(\nu)$ for Draco assuming three different DM profiles, NFW (red), Burkert (magenta), and D05 (cyan) [44]. The DM mass is $m_{\chi}=5 \mathrm{TeV}$, and the annihilation channel is $b \bar{b}$ with annihilation rate $\langle\sigma v\rangle=10^{-26} \mathrm{~cm}^{3} \mathrm{~s}^{-1}$. The values of diffusion coefficient and magnetic fields are $D_{0}=3 \times 10^{28} \mathrm{~cm}^{2} \mathrm{~s}^{-1}$ and $B=1 \mu \mathrm{G}$, respectively. Right panel: Same as left panel, but for $m_{\chi}=300 \mathrm{GeV}$.

The effects of diffusion coefficient and magnetic field on the final radio flux $S_{\nu}$ can be seen from Fig. 8. The radio flux corresponding to higher diffusion coefficient $\left(D_{0}=10^{30} \mathrm{~cm}^{2} \mathrm{~s}^{-1}\right)$ will be suppressed compared to the lower diffusion case $\left(D_{0}=3 \times 10^{28} \mathrm{~cm}^{2} \mathrm{~s}^{-1}\right)$ due to the escape of a large number of $e^{ \pm}$from the stellar object, as discussed above. This suppression is slightly larger in the lower-frequency region, since the number density $\frac{d n}{d E}$ decreases more in the low-energy regime (see Fig. 6). For, a constant $D_{0}$, say, $3 \times 10^{28} \mathrm{~cm}^{2} \mathrm{~s}^{-1}$, a relatively lower value of $B(0.1 \mu \mathrm{G})$ reduces the radio flux by about an order of magnitude in all frequency ranges. This is solely due to the fact that, although $\frac{d n}{d E}$ has similar values for different values of $B$ (1 and $0.1 \mu \mathrm{G})$, the synchrotron power spectrum $P_{\text {Synch }}$ decreases with a decrease in $B$ (as evident from Fig. 2). We further emphasize that, though this analysis assumes a NFW profile for Draco, the choice of other profiles such as Burkert [44,55] or Diemand et al. (2005) [56] (hereafter D05) [44] keeps our predictions similar, as can be seen in Fig. 9.

\section{HEAVY DM PARTICLES}

The radio flux obtained in terms of DM annihilation from a dSph crucially depends on the source function $Q_{e}(E, r)$ [Eq. (1)]. Let us try to explain why one can get a higher radio flux [obtained via $d n / d E$ through integration of $Q_{e}(E, r)$ ] for higher DM masses in some cases [37]. For this to happen, $Q_{e}$ corresponding to energetic $e^{ \pm}$is intuitively expected to go up for higher $m_{\chi}$. One may consider contributions of several components in the expression of $Q_{e}(E, r)$ :

(i) $\langle\sigma v\rangle$ : For a trans-TeV thermal $\mathrm{DM} \chi$, the factors affecting $\langle\sigma v\rangle$ are its mass $\left(m_{\chi}\right)$ and the effective couplings to SM particle pairs. While we are concerned here with the annihilation cross sections for $\chi$ within a dSph, one also needs consistency with the observed relic density [1]. In general, the expression for the relic density $\left(\Omega h^{2}\right)$ indicates inverse proportionality to $\langle\sigma v\rangle$, and $\frac{1}{m_{\chi}^{2}}$ occurs as the flux factor in the denominator of the latter [57]. Thus, a factor of $m_{\chi}^{2}$ occurs in the numerator. Therefore, $m_{\chi} \gtrsim 1 \mathrm{TeV}$ may make the relic density unacceptably large. One way to alleviate this is to ensure the possibility of resonant annihilation [57], as is possible in the MSSM through the participation of a pseudoscalar of appropriate mass [58]. This also serves to enhance the observed radio flux, as we shall see later. In addition, the annihilation of $\chi$ in the early Universe may involve channels other than those in a dSph, through coannihilation with particles spaced closely with it [58]. Once either of the above mechanisms is effective for the relevant particle spectrum, a trans-TeV DM particle remains consistent with all data including the relic density. This ensured, the pair annihilation of a trans-TeV $\mathrm{DM}$ particle in $\mathrm{dSphs}$ enables the production of relatively energetic $e^{ \pm}$pairs, which in turn reinforce the radio signals in the desired frequency range.

(ii) $N_{\text {pairs }}(r)\left(=\frac{\rho_{\chi}^{2}(r)}{2 m_{\chi}^{2}}\right)$ : The numerator is supplied by observation. For higher $m_{\chi}$, the denominator suppresses the number density of DM in the dSph. Thus, this term tends to bring down the flux for higher DM masses.

(iii) $\frac{d N_{f}^{e}(E)}{d E} B_{f}$ : This has to have a compensatory effect for higher mass if the suppression caused by the previously mentioned term has to be overcome. Of course, the branching fractions $B_{f}$ in different 

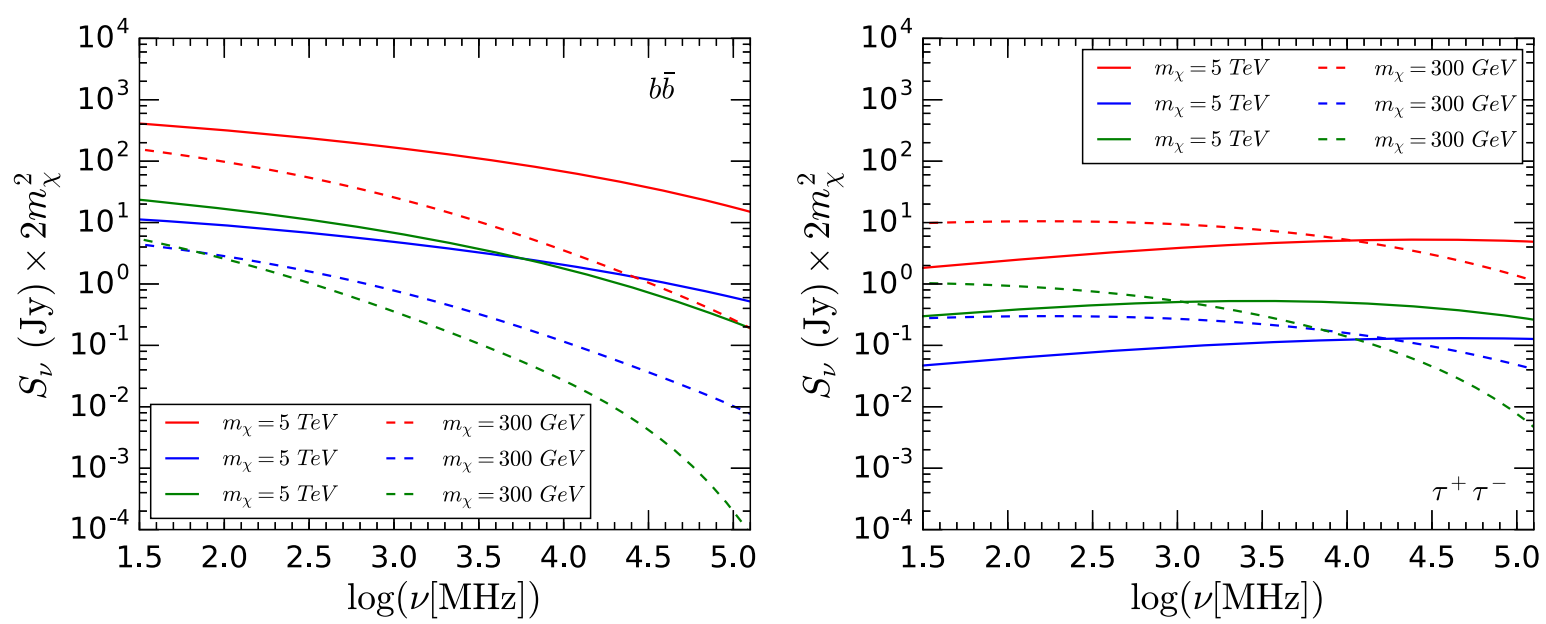

FIG. 10. $S_{\nu}(J y) \times 2 m_{\underline{\chi}}^{2}$ vs frequency $(\nu)$ plot for two DM masses, $300 \mathrm{GeV}$ (dashed lines) and $5 \mathrm{TeV}$ (solid lines), in two different annihilation channels, $b \bar{b}$ (left panel) and $\tau^{+} \tau^{-}$(right panel). Here, three different sets of astrophysical parameters have been considered, $D_{0}=3 \times 10^{28} \mathrm{~cm}^{2} \mathrm{~s}^{-1}, B=1 \mu \mathrm{G}$ (red); $D_{0}=10^{30} \mathrm{~cm}^{2} \mathrm{~s}^{-1}, B=1 \mu \mathrm{G}$ (blue); and $D_{0}=3 \times 10^{28} \mathrm{~cm}^{2} \mathrm{~s}^{-1}, B=0.1 \mu \mathrm{G}$ (green). The annihilation rate $\langle\sigma v\rangle=10^{-26} \mathrm{~cm}^{3} \mathrm{~s}^{-1}$ for all cases.

channels have a role. $d N^{e} / d E$ is often (though not always) higher for higher DM masses. This feature is universal at higher energies. This is basically responsible for a profusion of higher-energy electrons produced via cascades, after annihilation in any channel has been taken place. More discussion will follow on this point later. It should be noted that $d N^{e} / d E$ represents the probability that an electron (positron) produced from the annihilation of one pair of DM. This gets multiplied by the available number density of DM. Thus, for a comparable value of $B_{f}$ in two benchmark scenarios, higher $d N^{e} / d E$ along with higher $\langle\sigma v\rangle$ in the $\mathrm{dSph}$ (when that is indeed the case) can cause enhancement of $Q_{e}(E, r)$ for higher $m_{\chi}$.

Finally, the available electron energy distribution $\frac{d n}{d E}$ (which, convoluted with the synchrotron power spectrum, will yield the radio flux [i.e., Eqs. (8) and (9)]) is obtained by solving Eq. (3), which has a generic solution [Eq. (5)]. Therefore, higher values of the source function $\left[Q_{e}(E, r)\right]$ can produce higher radio flux at higher $m_{\chi}$.

As mentioned above, the annihilation of heavier DM particles will produce more energetic $e^{ \pm}$, as can be seen by comparing the upper left and upper right panels of Fig. 1, in which we have shown $d N^{e} / d E$ in different channels $(b \bar{b}$, $\tau^{+} \tau^{-}, W^{+} W^{-}$, and $\left.t \bar{t}\right)$. The lower panel shows the comparison of this $e^{ \pm}$spectrum for two DM masses $(300 \mathrm{GeV}$ and $5 \mathrm{TeV}$ ) arising from $b \bar{b}$ and $\tau^{+} \tau^{-}$annihilation channels. Note that the values of $d N^{e} / d E$ for $m_{\chi}=300 \mathrm{GeV}$ and $5 \mathrm{TeV}$ are differently ordered for the $\tau^{+} \tau^{-}$and $b \bar{b}$ annihilation channels. The spectrum for the $b \bar{b}$ channel is the steepest one, while $\tau^{+} \tau^{-}$is the flattest. In fact, $d N^{e} / d E$ for the $b \bar{b}$ channel is governed mostly by charged pion $\left(\pi^{ \pm}\right)$decay at various stages of cascade. For the $\tau^{+} \tau^{-}$ channel, on the other hand, there is a relative dominance of "prompt" electrons. There remains a difference in the degree of degradation in the two cases. Such degradation is reflected more in the low-energy part of the spectrum and leads to different energy distributions, which in turn is dependent on $m_{\chi}$ via the energy of the decaying $b$ or $\tau$. The reader is referred to Refs. [59-61] for more detailed explanations.

The presence of energetic $e^{ \pm}$in greater abundance, which is a consequence of higher $m_{\chi}$, enhances the resulting radio signal obtained through Eqs. (5) and (8). As a result of this, the final radio flux $S_{\nu}$ [Eq. (9)] gets a positive contribution for higher DM masses compared to that for relatively lower masses for most of the annihilation channels (especially, $b \bar{b}, W^{+} W^{-}$, and $t \bar{t}$ ). This can easily be seen if one compares the quantity $S_{\nu} \times 2 m_{\chi}^{2}$ (i.e., removing the effect of the multiplicative factor $\frac{1}{m_{\chi}^{2}}$ present in the expression of $N_{\text {pairs }}$ ) for two DM masses, $300 \mathrm{GeV}$ and $5 \mathrm{TeV}$, in the $b \bar{b}$ annihilation channel (left panel of Fig. 10). If one chooses same annihilation rate (e.g., $\langle\sigma v\rangle=10^{-26} \mathrm{~cm}^{3} \mathrm{~s}^{-1}$ ), the higher DM mass will give higher $S_{\nu} \times 2 m_{\chi}^{2}$ (mainly in the high-frequency region) for this channel. We have explicitly shown this for different choices of astrophysical parameters as indicated by different colors in the figure.

In scenarios in which $\langle\sigma v\rangle$ corresponding to a particular $m_{\chi}$ is calculated from the dynamics of the model, it can happen that for some particular benchmark the annihilation rate $(\langle\sigma v\rangle)$ is higher for larger $m_{\chi}$ (as mentioned earlier and will be discussed further in the next section). In those cases, larger $\langle\sigma v\rangle$ can at least partially compensate the effect of $\frac{1}{m_{\chi}^{2}}$ suppression (coming from $N_{\text {pairs }}$ ) for higher $m_{\chi}$. Thus, one can get higher radio fluxes $\left(S_{\nu}\right)$ for larger DM masses compared to the smaller one. 
For the $\tau^{+} \tau^{-}$channel, the situation is somewhat different. As we have already seen from the lower panel of Fig. 1, there is a large degradation of the $e^{ \pm}$flux $\left(d N^{e} / d E\right)$ in this channel in the low-energy region for higher $m_{\chi}$. This degradation in the source spectrum for higher $m_{\chi}$ will continue to be present in the equilibrium distribution $\frac{d n}{d E}$. After folding this density distribution with the power spectrum [see Eq. (8)], the final frequency distribution will be suppressed for higher $m_{\chi}$, mainly in the lowfrequency range. In the higher-frequency range, flux is still high for higher $m_{\chi}$, similar to that in other annihilation channel (e.g., $b \bar{b}$ ), because higher $m_{\chi}$ corresponds to higher electron distribution in the high-energy range. All these phenomena are evident from the red curves (solid and dashed) of the right panel in Fig. 10. Note that these curves are for the choice of astrophysical parameters $D_{0}=3 \times 10^{28} \mathrm{~cm}^{2} \mathrm{~s}^{-1}, B=1 \mu \mathrm{G}$. If one decreases the magnetic field $B$ to $0.1 \mu \mathrm{G}$ or increases the diffusion coefficient $D_{0}$ to $10^{30} \mathrm{~cm}^{2} \mathrm{~s}^{-1}$, the corresponding effect can be seen from the green and blue curves, respectively.

To summarize, one can expect high radio flux for trans$\mathrm{TeV}$ dark matter annihilation from a dSph, based on the following considerations:

(i) The high-mass DM candidate has to be consistent with relic density, for which $\langle\sigma v\rangle$ at freeze-out is the relevant quantity.

(ii) Such sizable $\langle\sigma v\rangle$ can still be inadequate in maintaining the observed relic density. Coannihilation channels may need to be available in these cases, though such coannihilation does not occur in a dSph. This, in turn, may necessitate a somewhat compressed trans-TeV spectrum.

(iii) The high-mass DM candidate should have appropriate annihilation channels which retain a higher population of $e^{ \pm}$. This not only offsets the suppression due to large $m_{\chi}$ but also enhances through the energy loss term $[b(E)]$ the $e^{ \pm}$density at energies low enough to contribute the radio observable at the SKA [33].

(iv) Higher magnetic fields will be more effective in producing synchrotron radiation by compensating the suppression caused by a large galactic diffusion coefficient. As we have checked through explicit calculation, this happens even after accounting for electromagnetic energy loss of the $e^{ \pm}$through synchrotron effects.

\section{DETECTABILITY CURVES AND FINAL RADIO FLUX}

The upcoming SKA radio telescope will play an important role in detecting DM-induced radio signal from dwarf spheroidal galaxies. Because of its large effective area and better baseline coverage, the SKA has a significantly higher surface brightness sensitivity compared to existing radio telescopes. Since the focus of this work is on the diffuse synchrotron signal from dSphs, we estimate the instrument sensitivity corresponding to the surface brightness and compare with the predicted signal. To calculate the sensitivity for a given source $\mathrm{dSph}$, we need to know the baseline distribution of the telescope. The sensitivity can also be affected by the properties of the sky around the source, e.g., whether there exists any other bright source in the field of view.

Since the detailed design of the SKA has yet to be finalized, it is difficult to estimate the noise in the direction of a given source. For our purpose, however, it is sufficient to estimate the approximate values of the sensitivity using the presently accepted baseline design. To do so, we make use of the documents provided on the SKA website [33]. The calculations presented in the document allow us to compute the expected sensitivity near zenith in a direction well away from the Galactic plane for all the frequencies relevant to the SKA, i.e., for both SKA-MID and SKALOW. These include contribution from the antenna receiver, the spillover, and the sky background (consisting of the cosmic microwave background, the galactic contribution, and also the atmospheric contribution). Note that we have assumed that all other observational systematics, such as the presence of other point sources in the field, the effect of the primary beam, the pointing direction dependence of the sky temperature, etc., are already corrected for and hence are not included in the calculation of the thermal noise.

From the above calculation, we find that typical values of the SKA surface brightness sensitivity in the frequency range $50 \mathrm{MHz}-50 \mathrm{GHz}$ for $100 \mathrm{~h}$ of observation time is $10^{-6}-10^{-7}$ Jy with a bandwidth of $300 \mathrm{MHz}[33,34,37]$. This may allow one to observe very low-intensity radio signal coming from ultrafaint dSphs. While comparing the predicted signal with the telescope sensitivity, we have assumed that the SKA field of view is larger than the galaxy sizes considered here, and hence all the flux from the galaxy will contribute to the detected signal. This assumption need not be true for the SKA precursors like the Murchison Widefield Array in which the effect of the primary beam needs to be accounted for while computing the expected signal [47].

To date, people do not have a clear understanding about either the DM particle physics model, which governs the production of the initial-stage $e^{ \pm}$spectrum, or the astrophysical parameters like the galactic diffusion coefficient $\left(D_{0}\right)$, magnetic field $(B)$, etc., which are responsible for the creation of radio synchrotron flux. Thus, in this analysis, we have constrained the DM parameter space, responsible for detecting the radio signal at SKA, for particular choices of astrophysical parameters which are on the conservative side for a typical dSph like Draco [44]. On the other hand, assuming some simplified DM model scenarios with trans-TeV DM masses, we have estimated the limits on the 

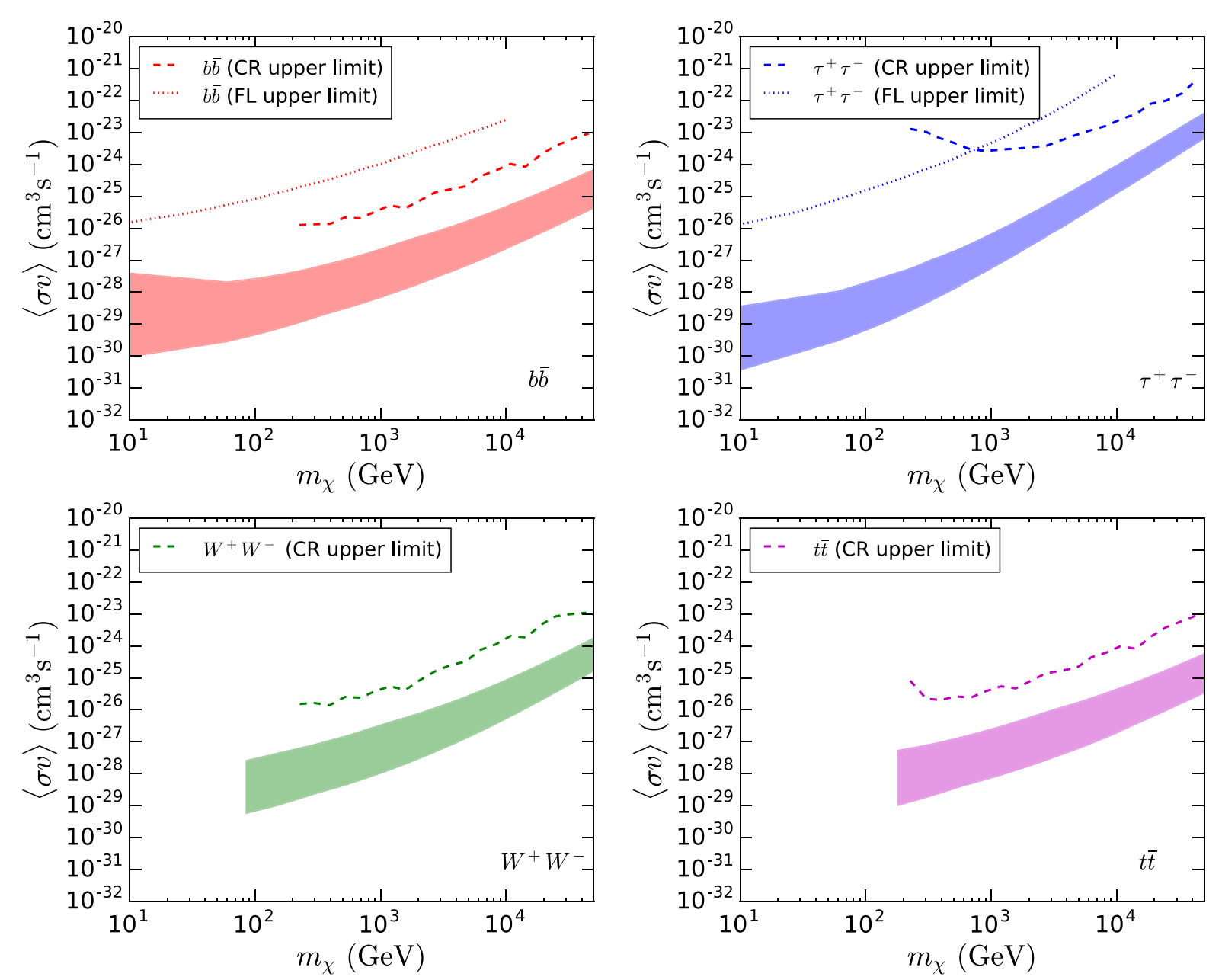

FIG. 11. Lower limits (colored bands) in the $\langle\sigma v\rangle-m_{\chi}$ plane to observe a radio signal from Draco dSph at SKA with $100 \mathrm{~h}$ of observation for various DM annihilation channels $\left(b \bar{b}\right.$, upper left; $\tau^{+} \tau^{-}$, upper right; $W^{+} W^{-}$, lower left; and $t \bar{t}$, lower right). For comparison, 95\% C.L. upper limits from CR antiproton observation (dashed lines) [28] and six years of FL data (dotted lines) [27] are also shown. The bands represent the variation of the magnetic field from $B=1 \mu \mathrm{G}$ (lower part of the bands) to a more conservative value $B=0.1 \mu \mathrm{G}$ (upper part of the bands). For all cases, the value of the diffusion coefficient $\left(D_{0}\right)$ is $3 \times 10^{28} \mathrm{~cm}^{2} \mathrm{~s}^{-1}$.

$B-D_{0}$ plane, required for the radio signal from Draco to be observed at SKA.

Figure 11 shows the minimum $\langle\sigma v\rangle$ required for any $m_{\chi}$ in four different annihilation channels $\left(b \bar{b}, \tau^{+} \tau^{-}, W^{+} W^{-}\right.$, and $t \bar{t}$ ) for detection of DM annihilation-induced radio signal from Draco with $100 \mathrm{~h}$ of observation at SKA assuming a typical bandwidth $300 \mathrm{MHz}$. The radio signal has been taken to be detectable when the observed flux in any frequency bin rises three times above the noise so as to ensure that the detection is statistically significant and is not affected by spurious noise features. We vary the DM mass over a wide range of $10 \mathrm{GeV}$ to $50 \mathrm{TeV}$, assuming $100 \%$ branching fraction $\left(B_{f}\right)$ in one annihilation channel at a time. The predictions here are for a conservative choice of the diffusion coefficient $\left(D_{0}=3 \times 10^{28} \mathrm{~cm}^{2} \mathrm{~s}^{-1}\right)$ [44]. The bands are due to the variation of the galactic magnetic field $B$ from $1 \mu \mathrm{G}$ (lower part of the band) to a more conservative value $0.1 \mu \mathrm{G}$ (upper part of the band). As expected, the minimum $\langle\sigma v\rangle$ required will be larger for lower magnetic fields as lower $B$ reduces the radio synchrotron frequency distribution $\left(S_{\nu}\right)$. These limits are the detection thresholds for SKA to observe radio signal from Draco. For $m_{\chi} \sim 1 \mathrm{TeV}$, this limit could be as low as $\langle\sigma v\rangle \sim 3 \times 10^{-29} \mathrm{~cm}^{3} \mathrm{~s}^{-1}$. For lower values of the diffusion coefficient such as $D_{0}=3 \times 10^{26} \mathrm{~cm}^{2} \mathrm{~s}^{-1}[30,31,52]$, the detectability threshold band comes down even further. Moreover, we have not considered any halo substructure contributions which are expected to enhance the radio flux [43] or lower the threshold limits even more. Along with these lower limits, we have also shown the modelindependent upper limits (in 95\% C.L.) on $\langle\sigma v\rangle$ in various channels from cosmic-ray (CR) antiproton observation (dashed curves) [28] and from six years of Fermi LAT (FL) $\gamma$-ray data (dotted curves) [27]. Note that the upper bounds from cosmic-ray antiproton observations are the strongest ones. For each annihilation channel, the area 
TABLE I. Lightest neutralino mass $\left(m_{\chi_{1}^{0}}\right)$ and its pair annihilation rate $(\langle\sigma v\rangle)$ inside a dSph along with branching fractions ( $B_{f}$ in percent) in different annihilation channels for the selected MSSM benchmark points from Ref. [37]. The lightest neutralino is the DM candidate $\left(m_{\chi_{1}^{0}}=m_{\chi}\right)$.

\begin{tabular}{lccc}
\hline \hline Model & Annihilation channel $\left(B_{f}\right)$ & $m_{\chi_{1}^{0}}(\mathrm{GeV})$ & $\langle\sigma v\rangle\left(10^{-26} \mathrm{~cm}^{3} \mathrm{~s}^{-1}\right)$ \\
\hline A1a & $b \bar{b}(85 \%), \tau^{+} \tau^{-}(14 \%)$ & 1000.6 & 0.27 \\
B2a & $b \bar{b}(76 \%), \tau^{+} \tau^{-}(15 \%), W^{+} W^{-}(3 \%), t \bar{t}(3 \%), Z Z(2.8 \%)$ & 3368.0 & 1.19 \\
E & $b \bar{b}(79.1 \%), \tau^{+} \tau^{-}(18.3 \%), t \bar{t}(2.5 \%)$ & 8498.0 & 9.12 \\
\hline \hline
\end{tabular}

bounded by the upper and lower limits represents the region in the $\langle\sigma v\rangle-m_{\chi}$ plane which can be probed or constrained by SKA with $100 \mathrm{~h}$ of observation. It is clear from the figure that, even with conservative choices of astrophysical parameters for $m_{\chi} \approx 50 \mathrm{TeV}$, there are significantly large regions of the parameter space which can be probed in SKA. In such extreme cases, it is, of course, necessary to have a dark sector that allows high coannihilation rates so that the observed relic density bound is not excluded.

In the context of model-dependent analysis, three benchmark points, named models A1a, B2a, and E, from our earlier work [37] have been considered here. These benchmarks correspond to the MSSM scenario in which the lightest neutralino $\left(\chi_{1}^{0}\right)$ is the DM candidate $(\chi)$. Table I contains the possible annihilation channels with branching fractions, DM masses $\left(m_{\chi_{1}^{0}}\right)$, and annihilation rates $(\langle\sigma v\rangle)$ calculated in those benchmark points. All these quantities have been calculated using the publicly available package MICROMEGAs $[49,62]$. Masses of neutralino and all other supersymmetric particles in these three cases are in the trans-TeV range. All of these benchmarks produce relic densities within the expected observational limits $[1,63,64]$ and satisfy constraints coming from direct DM searches [2,3], collider study [65], lightest neutral Higgs mass measurements [66], and other experiments $[67,68]$. These benchmarks have been discussed in further detail in Ref. [37]. Figure 12 shows the detectability of these benchmarks for Draco dSph in SKA after $100 \mathrm{~h}$ of observations. The predicted flux in each case falls within the area which is still allowed by the data and is at least three times above the observation threshold of SKA. The upper limits here are taken from cosmic-ray antiproton observation at $95 \%$ C.L [28], and the lower limits have been calculated for $D_{0}=3 \times 10^{28} \mathrm{~cm}^{2} \mathrm{~s}^{-1}$ and $B=1 \mu \mathrm{G}$. Along with these, we have indicated the total $\langle\sigma v\rangle$ (listed in the third column of Table I) by different points for each benchmark. It should be noted that each such benchmark allows more than one annihilation channel. Consistently, the cross sections are obtained by taking a sum over different channels appropriately weighted by the branching ratios:

$$
\langle\sigma v\rangle=\sum_{f}\langle\sigma v\rangle_{f} B_{f}
$$

It is clear that all these high-mass cases, which are allowed by cosmic-ray antiproton data, can easily be probed in SKA with $100 \mathrm{~h}$ of observations. This is due to the following reasons:

(i) $\langle\sigma v\rangle$ is more effective in offsetting $\frac{1}{m_{x}^{2}}$ suppression [present in the expression for number density of DM pair, Eq. (1)] partially, though not fully.

(ii) A greater abundance of high-energy $e^{ \pm}$is created by high $m_{\chi}$. This, via electromagnetic energy loss driven by the term proportional to $b(E)$ in Eq. (3), generates a bigger flux of radio synchrotron emission, as evinced in the expression for $J_{\text {Synch }}(\nu, r)$ [Eq. (8)].

(iii) The cases in which one predicts more intense radio flux for higher $m_{\chi}$ have DM annihilation mostly in the $b \bar{b}$ channels, as against the $\tau^{+} \tau^{-}$channel. The corresponding cascade branching ratios as well as the three-body decay matrix elements and their

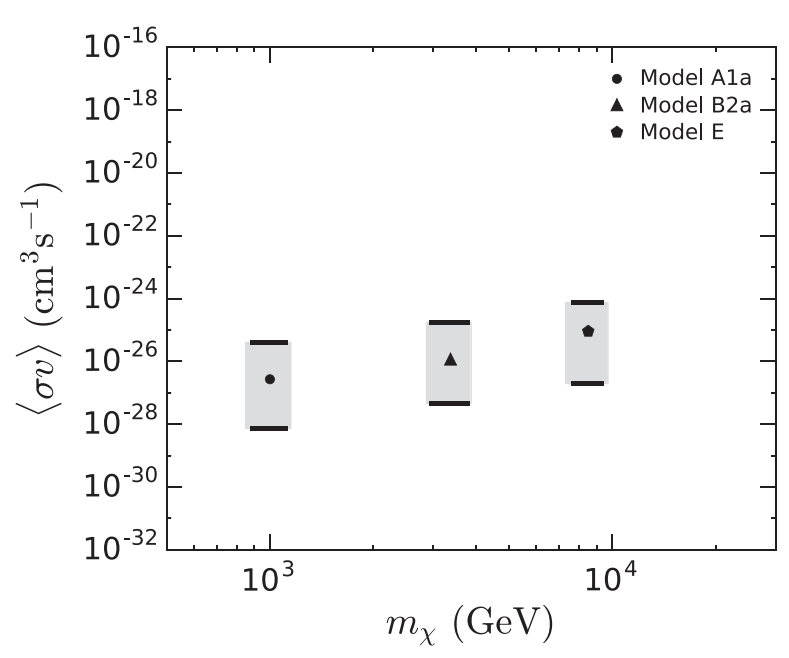

FIG. 12. Location of various MSSM benchmark points (models A1a, B2a, and E, listed in Table I) from Ref. [37] in the $\langle\sigma v\rangle-m_{\chi}$ plane. The upper bars represent the $95 \%$ C.L. upper limits on $\langle\sigma v\rangle$ corresponding to these benchmark points from CR antiproton observation [28]. The lower bars show the minimum $\langle\sigma v\rangle$ required for those benchmark points for the observation of radio flux from Draco dSph at SKA with $100 \mathrm{~h}$ of observations. The diffusion coefficient and the magnetic field have been assumed as $D_{0}=3 \times 10^{28} \mathrm{~cm}^{2} \mathrm{~s}^{-1}$ and $B=1 \mu \mathrm{G}$, respectively. 

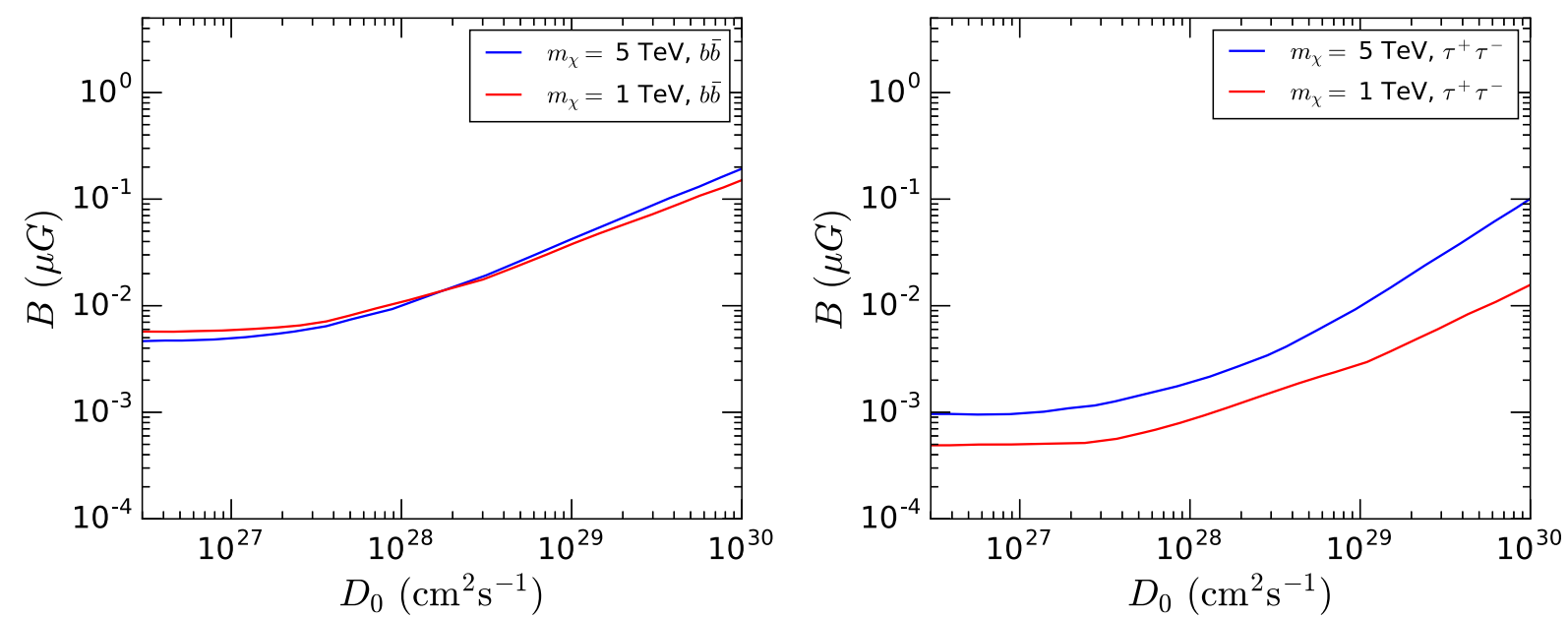

FIG. 13. Limits in the $B-D_{0}$ plane to observe a radio signal from Draco dSph with $100 \mathrm{~h}$ of observation at SKA for $m_{\chi}=5 \mathrm{TeV}$ (blue curves) and $1 \mathrm{TeV}$ (red curves). Annihilation channels are $b \bar{b}$ (left panel) and $\tau^{+} \tau^{-}$(right panel). The DM annihilation rate ( $\langle\sigma v\rangle$ ) in each case has been assumed to be the 95\% C.L. upper limit as obtained from cosmic-ray antiproton observation [28].

energy integration limits are responsible for bigger radio flux. ${ }^{4}$

Figure 11 shows how the detectability threshold at the SKA varies with the galactic magnetic field $B$, for a fixed value of $D_{0}$. However, the values of these two parameters for the dSphs are quite uncertain. In view of this, it is also important to find out which regions in the astrophysical parameter space are within the scope of the SKA with $100 \mathrm{~h}$ of observation, when both $B$ and $D_{0}$ vary over substantial ranges. The detectability of the signal in the $B-D_{0}$ space is shown in Fig. 13 for some illustrative DM scenarios. These correspond to DM masses of 1 (red curve) and 5 (blue curve) TeV. Cases in which the dominant annihilation channel is $b \bar{b}$ are shown in the left panel, while the curves on the right panel capture the corresponding situations with $\tau^{+} \tau^{-}$as the main channel. The solid line of each color corresponds to the maximum value of $\langle\sigma v\rangle$ for the chosen $m_{\chi}$, consistent with the cosmic-ray upper limit. All points above and on the left of the curve correspond to the combinations of $B$ and $D_{0}$ that make the radio signals detectable over $100 \mathrm{~h}$ of observations with SKA. Points for higher $B$ in this region correspond to models that are detectable even with lower values of $\langle\sigma v\rangle$. Similarly, the detectable models having progressively lower $\langle\sigma v\rangle$ are arrived at, as one moves to lower $D_{0}$ for a fixed value of $B$. It is evident from both the $b \bar{b}$ and $\tau^{+} \tau^{-}$channels (chosen for illustrations in Fig. 13) that consistent values of $\langle\sigma v\rangle$ can lead to detectability with $100 \mathrm{~h}$ at SKA, for $B \gtrsim 5 \times$ $10^{-4} \mu \mathrm{G}$ for $D_{0} \sim 10^{27} \mathrm{~cm}^{2} \mathrm{~s}^{-1}$. On the other hand, for larger values of $D_{0} \sim 10^{30} \mathrm{~cm}^{2} \mathrm{~s}^{-1}$, we need $B \gtrsim 10^{-2} \mu \mathrm{G}$ for the signal to be detectable.

\footnotetext{
${ }^{4}$ Such effects can, in principle, be also expected if the $t \bar{t}, W^{+} W^{-}$branching ratios dominate.
}

Finally, we show in Fig. 14 the final radio fluxes in two of the MSSM benchmarks listed in Table I. For illustration, we have taken the benchmark points A1a $\left(m_{\chi} \sim 1 \mathrm{TeV}\right)$ and $\mathrm{E}\left(m_{\chi} \sim 8.5 \mathrm{TeV}\right)$. The annihilation is $b \bar{b}$ dominated for both cases. The yellow band here represents the SKA sensitivity [33], with a bandwidth of $300 \mathrm{MHz}$. The band is due to the variation of the observation time from 10 (upper part of the band) to $100 \mathrm{~h}$ (lower part of the band) [33,37]. The choice of the diffusion coefficient and the magnetic field is on the conservative side $\left(D_{0}=3 \times 10^{28} \mathrm{~cm}^{2} \mathrm{~s}^{-1}\right.$ and $B=1 \mu \mathrm{G})$. We have already shown the detectability of these benchmarks in Fig. 12. From this figure, we can see

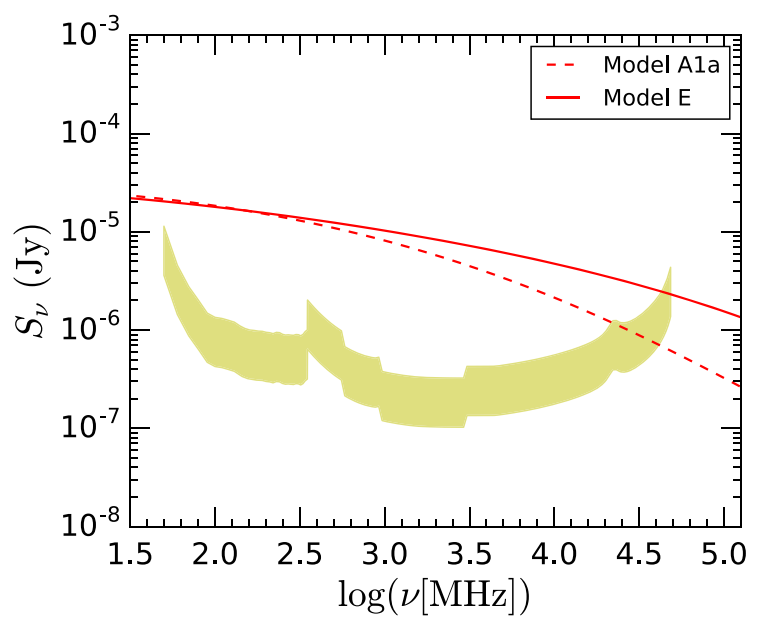

FIG. 14. Radio synchrotron flux $\left(S_{\nu}\right)$ vs frequency $(\nu)$ plot for Draco dSph using two MSSM benchmark points A1a and E from Table I. The yellow shaded band denotes the SKA sensitivity corresponding to the variation of observation time from 10 (upper part of the band) to $100 \mathrm{~h}$ (lower part of the band) $[33,37]$. The diffusion coefficient is $D_{0}=3 \times 10^{28} \mathrm{~cm}^{2} \mathrm{~s}^{-1}$, and the magnetic field is $B=1 \mu \mathrm{G}$. 
that, even for $10 \mathrm{~h}$ of observation, these high- $m_{\chi}$ benchmarks can easily be observed in SKA over most of its frequency range.

One important feature of Fig. 14 is that, in a wide range of frequency (300 MHz-50 GHz), suitable for SKA, $S_{\nu}$ for the very high-mass case (model $\mathrm{E}$ ) is higher than that for the low-mass case (model A1a). Though the DM mass in model $\mathrm{E}$ is larger, the annihilation rate in this case is higher (approximately $9 \times 10^{-26} \mathrm{~cm}^{3} \mathrm{~s}^{-1}$ ) than in model A1a (approximately $3 \times 10^{-27} \mathrm{~cm}^{3} \mathrm{~s}^{-1}$ ). In general, $\langle\sigma v\rangle$ should have a $\frac{1}{m_{\chi}^{2}}$ suppression, because of the energies of the colliding DM particles [57]. In spite of that, model E (higher $m_{\chi}$ ) has greater $\langle\sigma v\rangle^{5}$ than model A1a (lower $m_{\chi}$ ) mainly due to the closer proximity to an s-channel resonance mediated bya $C P$-odd pseudoscalar in the annihilation process like $\chi_{1}^{0} \chi_{1}^{0} \rightarrow b \bar{b}$. For detailed information, the reader is referred to Ref. [37].

As discussed earlier, the higher value of $\langle\sigma v\rangle$ partially offsets the effect of larger DM mass, and consequently, we get $\frac{\langle\sigma v\rangle}{m_{\chi}^{2}}$ [which appears in the source function, Eq. (1)] for model A1a and model E as 2.7 and 1.26 (in units of $10^{-33} \mathrm{GeV}^{-2} \mathrm{~cm}^{3} \mathrm{~s}^{-1}$ ), respectively, though the latter model has larger $m_{\chi}$ (approximately 8.5 TeV) compared to the former (approximately $1 \mathrm{TeV}$ ). Thus, a 72-fold suppression due to $m_{\chi}^{2}$ results in a suppression just by a factor of approx. 2 at the level of $\frac{\langle\sigma v\rangle}{m_{\chi}^{2}}$. Also, note that the $b \bar{b}$ annihilation channel dominates for both models. These observations, together with the discussion in Sec. IV, and the contents of Fig. 10, explain a higher-mass DM particle generating higher radio flux for scenarios in which the $b \bar{b}$ annihilation channel dominates over $\tau^{+} \tau^{-}$.

\section{CONCLUSION}

The aim of this paper is to show that radio signal arising from a high-mass (trans-TeV) WIMP DM can be detectable as radio synchrotron flux from a dSph, to be recorded by the upcoming SKA telescope. We have analyzed not only the particle physics aspects of DM annihilation and subsequent cascades leading to $e^{ \pm}$pairs but have also included the relevant astrophysical processes the electrons/ positrons pass through before emitting radio waves, upon acceleration by the galactic magnetic field. We have set out to identify the mechanism whereby a trans-TeV DM candidate can thus be visible in the radio search. We found that in the SKA frequency range enhancement of the radio flux for this case is possible mainly due to the following reasons:

\footnotetext{
${ }^{5}$ A higher value of $\langle\sigma v\rangle$ for higher $m_{\chi}$ is also needed to keep the relic density under the observed limit, even when there is scope of coannihilation in the early Universe.
}

(i) A larger cross section or annihilation rate (required to maintain relic density under the observed limit for a trans-TeV DM) facilitated by the dynamics of the particle physics model. This helps compensate the $\frac{1}{m_{\chi}^{2}}$ suppression due to large DM mass.

(ii) The presence of energetic $e^{ \pm}$in the DM annihilation spectrum in greater abundance. This partially reduces the $\frac{1}{m_{\chi}^{2}}$ suppression effect and, on the other hand, enhances, through the energy loss term, the electron-positron density at low energies, which helps to produce large radio flux.

(iii) The dominance of the annihilation channel $b \bar{b}$, which yields a comparatively larger abundance of $e^{ \pm}$in all of the energy range of the spectrum produced by DM annihilation.

Simultaneously, the effect of various astrophysical parameters [e.g., $D_{0}, b(E)$, and $\left.B\right]$ on the radio synchrotron flux produced from the annihilation of a trans-TeV DM particle has been studied in detail.

Using SKA sensitivity, we have drawn the limits in the $\langle\sigma v\rangle-m_{\chi}$ plane to observe radio flux from Draco with $100 \mathrm{~h}$ of observation. We found that these limits are much stronger than the previously obtained bounds on $\langle\sigma v\rangle$ from Fermi-LAT $\gamma$-ray [27] or AMS-02 cosmic-ray antiproton observation [28]. Even for a conservative choice of astrophysical parameters $\left(D_{0}=3 \times 10^{28} \mathrm{~cm}^{2} \mathrm{~s}^{-1}\right.$ and $B=1 \mu \mathrm{G}$ ), we found that these limits can go as low as $\langle\sigma v\rangle \sim 3 \times 10^{-29} \mathrm{~cm}^{3} \mathrm{~s}^{-1}$ for a $m_{\chi} \sim 1 \mathrm{TeV}$. This indicates toward a large region of WIMP parameter space which can be probed through the upcoming SKA. Along with these, we have also shown the limits in the $B-D_{0}$ plane. We found that for a DM mass in the trans-TeV range, magnetic field as low as $B \sim 10^{-3} \mu \mathrm{G}$ and diffusion coefficient as high as $D_{0} \sim 10^{30} \mathrm{~cm}^{2} \mathrm{~s}^{-1}$ are well enough to produce radio flux above SKA sensitivity in $100 \mathrm{~h}$ of observation time.

Taking the MSSM as an illustration, we have shown that benchmark points with lightest neutralino masses $\left(m_{\chi_{1}^{0}}\right)$ approximately $1-8 \mathrm{TeV}$, which satisfy all the constraints from observed relic density, direct DM searches, and collider searches, can lead to detectable signals at the SKA with $100 \mathrm{~h}$ observation, even with a conservative choice of $B$ and $D_{0}$. We have illustrated how the effects mentioned earlier can lead to a larger radio flux for a highmass DM benchmark point compared to a low-mass case, thus establishing the credibility of the search for heavy DM through radio observation in SKA. ${ }^{6}$

\footnotetext{
${ }^{6}$ Recently, we came to know of Ref. [69], in which a similar study has been carried out for some different scenarios. While the broad conclusions have some similarity, our conclusions are a little more optimistic, since we have predicted detectability with $100 \mathrm{~h}$ of observation, with realistic benchmarks. One of the reasons that have led to the more positive predictions is the inclusion of the $b \bar{b}$ annihilation channel in our study.
} 


\section{ACKNOWLEDGMENTS}

The work of A.K. and B. M. was supported by the funding available from the Department of Atomic Energy, Government of India, for the Regional Centre for
Accelerator-based Particle Physics (RECAPP), HarishChandra Research Institute. T. R. C. and S. M. acknowledge the hospitality of RECAPP during the formulation of the project.
[1] P. A. R. Ade et al. (Planck Collaboration), Astron. Astrophys. 571, A16 (2014).

[2] E. Aprile et al. (XENON Collaboration), Phys. Rev. Lett. 119, 181301 (2017).

[3] D. S. Akerib et al. (LUX Collaboration), Phys. Rev. Lett. 118, 251302 (2017).

[4] M. Carena, J. Osborne, N. R. Shah, and C. E. M. Wagner, Phys. Rev. D 100, 055002 (2019).

[5] J. Ellis, in Proceedings of the 38th International Symposium on Physics in Collision (PIC 2018) Bogot, Colombia, 2018 (2018), https://arxiv.org/abs/1810.11263.

[6] T. Abe et al. (LHC Dark Matter Working Group), Phys. Dark Universe, 100351 (2019).

[7] L. Shchutska (CMS Collaboration), Nucl. Part. Phys. Proc. 273-275, 656 (2016).

[8] S. P. Martin, Adv. Ser. Dir. High Energy Phys. 18, 1 (1998).

[9] The ATLAS Collaboration, Tech. Rep. ATL-PHYS-PUB2014-010, CERN, Geneva, 2014, https://cds.cern.ch/record/ 1735031.

[10] CMS Collaboration, Tech. Rep. CMS-PAS-FTR-13-014, CERN, Geneva, 2013, https://cds.cern.ch/record/1607141? $\ln =$ en.

[11] The ATLAS Collaboration, Tech. Rep. ATL-PHYS-PUB2013-011, CERN, Geneva, 2013, http://cdsweb.cern.ch/ record/1604505.

[12] D. Suematsu, Eur. Phys. J. C 56, 379 (2008).

[13] E. Morgante, in Proceedings of the 13th Rencontres $d u$ Vietnam: Exploring the Dark Universe Quy Nhon, Vietnam, 2017 (2017), https://arxiv.org/abs/1711.00767.

[14] T. Daylan, D. P. Finkbeiner, D. Hooper, T. Linden, S. K. N. Portillo, N. L. Rodd, and T. R. Slatyer, Phys. Dark Universe 12, 1 (2016).

[15] F. Calore, I. Cholis, C. McCabe, and C. Weniger, Phys. Rev. D 91, 063003 (2015).

[16] A. Kar, S. Mitra, B. Mukhopadhyaya, and T. R. Choudhury, J. Cosmol. Astropart. Phys. 02 (2018) 045.

[17] A. Kar, S. Mitra, B. Mukhopadhyaya, and T. R. Choudhury, arXiv:1711.09069.

[18] C. Karwin, S. Murgia, T. M. P. Tait, T. A. Porter, and P. Tanedo, Phys. Rev. D 95, 103005 (2017).

[19] G. Beck and S. Colafrancesco, J. Cosmol. Astropart. Phys. 10 (2017) 007.

[20] A. Achterberg, M. van Beekveld, W. Beenakker, S. Caron, and L. Hendriks, J. Cosmol. Astropart. Phys. 12 (2015) 013.

[21] A. Hektor, M. Raidal, A. Strumia, and E. Tempel, Phys. Lett. B 728, 58 (2014).

[22] Y. Bai, J. Berger, and S. Lu, Phys. Rev. D 97, 115012 (2018).
[23] I. Cholis, T. Linden, and D. Hooper, Phys. Rev. D 99, 103026 (2019).

[24] M.-Y. Cui, X. Pan, Q. Yuan, Y.-Z. Fan, and H.-S. Zong, J. Cosmol. Astropart. Phys. 06 (2018) 024.

[25] M. Ackermann et al. (Fermi-LAT Collaboration), Astrophys. J. 840, 43 (2017).

[26] A. Albert et al. (Fermi-LAT, DES Collaborations), Astrophys. J. 834, 110 (2017).

[27] M. Ackermann et al. (The Fermi-LAT Collaboration), Phys. Rev. Lett. 115, 231301 (2015).

[28] A. Cuoco, J. Heisig, M. Korsmeier, and M. Krmer, J. Cosmol. Astropart. Phys. 04 (2018) 004.

[29] M. Regis, L. Richter, and S. Colafrancesco, J. Cosmol. Astropart. Phys. 07 (2017) 025.

[30] A. Natarajan, J. E. Aguirre, K. Spekkens, and B. S. Mason, arXiv:1507.03589.

[31] A. Natarajan, J. B. Peterson, T. C. Voytek, K. Spekkens, B. Mason, J. Aguirre, and B. Willman, Phys. Rev. D 88, 083535 (2013).

[32] K. Spekkens, B. S. Mason, J. E. Aguirre, and B. Nhan, Astrophys. J. 773, 61 (2013).

[33] R. Braun et al., https://astronomers.skatelescope.org/wpcontent/uploads/2017/10/SKA-TEL-SKO-0000818-01_ SKA1_Science_Perform.pdf/ (2017).

[34] S. Colafrancesco, M. Regis, P. Marchegiani, G. Beck, R. Beck, H. Zechlin, A. Lobanov, and D. Horns, Proc. Sci., AASKA14 (2015) 100 [arXiv:1502.03738].

[35] M. Cirelli and M. Taoso, J. Cosmol. Astropart. Phys. 07 (2016) 041.

[36] M. Regis, S. Colafrancesco, S. Profumo, W. J. G. de Blok, M. Massardi, and L. Richter, J. Cosmol. Astropart. Phys. 10 (2014) 016.

[37] A. Kar, S. Mitra, B. Mukhopadhyaya, and T. R. Choudhury, Phys. Rev. D 99, 021302(R) (2019).

[38] L. E. Strigari, J. S. Bullock, M. Kaplinghat, J. D. Simon, M. Geha, B. Willman, and M. G. Walker, Nature (London) 454, 1096 (2008).

[39] M. Geha, B. Willman, J. D. Simon, L. E. Strigari, E. N. Kirby, D. R. Law, and J. Strader, Astrophys. J. 692, 1464 (2009).

[40] S. Colafrancesco, S. Profumo, and P. Ullio, Astron. Astrophys. 455, 21 (2006).

[41] A. McDaniel, T. Jeltema, S. Profumo, and E. Storm, J. Cosmol. Astropart. Phys. 09 (2017) 027.

[42] T. Linden, S. Profumo, and B. Anderson, Phys. Rev. D 82, 063529 (2010).

[43] G. Beck and S. Colafrancesco, J. Cosmol. Astropart. Phys. 05 (2016) 013.

[44] S. Colafrancesco, S. Profumo, and P. Ullio, Phys. Rev. D 75, 023513 (2007). 
[45] A. Geringer-Sameth, S. M. Koushiappas, and M. Walker, Astrophys. J. 801, 74 (2015).

[46] J. Choquette, Phys. Rev. D 97, 043017 (2018).

[47] A. Kar, S. Mitra, B. Mukhopadhyaya, T. R. Choudhury, and S. Tingay, Phys. Rev. D 100, 043002 (2019).

[48] J. F. Navarro, C. S. Frenk, and S. D. M. White, Astrophys. J. 462, 563 (1996).

[49] G. Blanger, F. Boudjema, A. Pukhov, and A. Semenov, http://lapth.cnrs.fr/micromegas/.

[50] G. Belanger, F. Boudjema, P. Brun, A. Pukhov, S. RosierLees, P. Salati, and A. Semenov, Comput. Phys. Commun. 182, 842 (2011).

[51] E. Storm, T. E. Jeltema, M. Splettstoesser, and S. Profumo, Astrophys. J. 839, 33 (2017).

[52] T. E. Jeltema and S. Profumo, Astrophys. J. 686, 1045 (2008).

[53] F. Donato, N. Fornengo, D. Maurin, P. Salati, and R. Taillet, Phys. Rev. D 69, 063501 (2004).

[54] M. Regis, L. Richter, S. Colafrancesco, S. Profumo, W. J. G. de Blok, and M. Massardi, Mon. Not. R. Astron. Soc. 448, 3747 (2015).

[55] A. Burkert, IAU Symp. 171, 175 (1996); Astrophys. J. 447, L25 (1995).

[56] J. Diemand, M. Zemp, B. Moore, J. Stadel, and M. Carollo, Mon. Not. R. Astron. Soc. 364, 665 (2005).
[57] P. Gondolo and G. Gelmini, Nucl. Phys. B360, 145 (1991).

[58] R. C. Gilmore, Phys. Rev. D 76, 043520 (2007).

[59] S. Colafrancesco and B. Mele, Astrophys. J. 562, 24 (2001).

[60] S. Rudaz and F. W. Stecker, Astrophys. J. 325, 16 (1988).

[61] M. Kamionkowski and M. S. Turner, Phys. Rev. D 43, 1774 (1991).

[62] G. Belanger, F. Boudjema, A. Pukhov, and A. Semenov, Comput. Phys. Commun. 149, 103 (2002).

[63] J. Harz, B. Herrmann, M. Klasen, K. Kovarik, and P. Steppeler, Phys. Rev. D 93, 114023 (2016).

[64] M. Klasen, K. Kovarik, and P. Steppeler, Phys. Rev. D 94, 095002 (2016).

[65] M. Aaboud et al. (ATLAS Collaboration), J. High Energy Phys. 01 (2018) 055.

[66] B. C. Allanach, A. Djouadi, J. L. Kneur, W. Porod, and P. Slavich, J. High Energy Phys. 09 (2004) 044.

[67] R. Aaij et al. (LHCb Collaboration), Phys. Rev. Lett. 118, 191801 (2017).

[68] S. Sandilya (Belle-II Collaboration), Proc. Sci., CKM2016 (2017) 080 [arXiv:1706.01027].

[69] J. A. R. Cembranos, Ã. De La Cruz-Dombriz, V. Gammaldi, and M. Mndez-Isla, Phys. Dark Universe 27, 100448 (2020). 